\title{
The Population Genetics of Ploidy Change in Fungi
}

Aleeza C Gerstein ${ }^{* 1}$ \& Nathaniel Sharp*2

* equal contribution

${ }^{1}$ Dept. of Microbiology, Dept. of Statistics, University of Manitoba Canada

2 Dept. of Genetics, University of Wisconsin-Madison USA

Correspondence: aleeza.gerstein@umanitoba.ca

\section{Abstract}

Ploidy is a significant type of genetic variation, describing the number of chromosome sets per cell. Ploidy evolves in natural populations, clinical populations, and lab experiments, particularly in fungi. Despite a long history of theoretical work on this topic, predicting how ploidy will evolve has proven difficult, as it is often unclear why one ploidy state outperforms another. Here, we review what is known about contemporary ploidy evolution in diverse fungal species through the lens of population genetics. As with typical genetic variants, ploidy evolution depends on the rate that new ploidy states arise by mutation, natural selection on alternative ploidy states, and random genetic drift. However, ploidy variation also has unique impacts on evolution, with the potential to alter chromosomal stability, the rate and patterns of point mutation, and the nature of selection on all loci in the genome. We discuss how ploidy evolution depends on these general and unique factors and highlight areas where additional experimental evidence is required to comprehensively explain the ploidy transitions observed in the field and the lab.

\section{Keywords}

selection; mutation; genetic drift; adaptation; ploidy drive; genome instability

\section{Introduction: Ploidy change}

A fundamental genetic feature of all organisms is ploidy, the number of homologous chromosome sets in a given cell. In species that undergo sexual reproduction, regulated 
processes predictably increase and decrease ploidy via syngamy and meiosis. Ploidy can also change over time within a population of cells through errors in mitosis (referred to as endoreduplication, endomitosis, or endopolyploidy; Davoli and de Lange 2011; Fox and Duronio 2013). These developmental processes that produce cells of increased ploidy occur among certain cell types and tissues in plants and animals (e.g., Davoli and de Lange 2011; Fox and Duronio 2013; Edgar, Zielke and Gutierrez 2014). Unregulated changes in ploidy are also possible and increasingly appreciated as common occurrences for some organisms. Cumulatively these asexual increases and decreases in ploidy are referred to as the "parasexual cycle" (Bennett and Johnson 2003).

Despite this variability, it is practical to define the "baseline ploidy" of a given population as the state of the majority of somatic cells, or the predominant state across the life cycle. Here we will define baseline ploidy as the copy number of the majority of chromosomes. Regardless of ploidy, cells can be euploid, containing an equal copy number of all chromosomes, or aneuploid, where chromosomes are present in unequal numbers. We will use the term "ploidy" only to refer to changes in baseline ploidy. Our focus here is on ploidy changes that do not involve more than one taxon (i.e., autopolyploidy), but allopolyploidy is an important evolutionary pathway in its own regard and has played a critical role in the evolution of many fungal clades (Gabaldón 2020).

Given the developmental lability of ploidy, it is perhaps not surprising that the baseline ploidy level varies widely among organisms. Shifts have evolved many times throughout evolution, e.g., in fungi (Albertin and Marullo 2012), plants (Wood et al. 2009) and animals (Gregory and Mable 2008), with ancestral whole-genome duplications appearing throughout the eukaryotic kingdom. However, our understanding of the drivers and consequences of long-term ploidy variation remains incomplete, despite long-standing interest in the topic from historical evolutionary luminaries (Haldane 1933; Stebbins 1947; Ohno 1970) and an understanding among contemporary biologists of the importance of ploidy in ecological and evolutionary processes (e.g., Madlung 2013; Ramsey and Ramsey 2014, and references therein).

While ploidy shifts share some features with "typical" mutations, these events are not always included in discussions on the variety of possible genetic changes. Perhaps this is because some ploidy shifts occur in a coordinated fashion, such that an unregulated ploidy shift could produce a ploidy state that is already frequent, unlike most other mutations. Arguably, all ploidy 
changes, including syngamy and meiosis, might qualify as mutations in the strict sense, since these events alter the allelic and structural context of the genome. Setting aside these semantic considerations, we will argue that many aspects of ploidy evolution are best understood by treating unregulated ploidy shifts as mutations.

Our goal here is to consider the genetics of ploidy shifts in a way that is analogous to the genetics of other types of mutant alleles while highlighting aspects of ploidy shifts that make this type of genetic change unique. In short, we examine ploidy shifts through the lens of population genetics: there is some rate of spontaneous transition between states, some consequence of such shifts for organismal fitness, and some likelihood that a given change will become "fixed" in a population of a given size, given the effectiveness of natural selection, with the potential for the environment to affect all of the above. Ploidy shifts have the potential to be beneficial, neutral, or deleterious, depending on the environment and genetic background in which they occur, just like typical mutations. The rate that ploidy shifts arise and spread depends on the same parameters that influence the evolutionary trajectory of all alleles: selection, mutation, and genetic drift.

In applying population genetic principles to the study of spontaneous ploidy changes, we should distinguish between evolution and adaptation. Evolution means a change in the frequency of some genetic variant over time, regardless of the fitness effects of that variant. Mutation, selection, gene flow and genetic drift all contribute to evolution. Adaptation is a type of evolution where natural selection acts to increase the frequency of favourable genetic variants, improving the mean fitness of the population. This critical distinction is often not made in the literature on ploidy shifts, where genetic changes are sometimes assumed to be adaptive without evidence.

We focus our discussion on the fungal kingdom —an often neglected, yet fascinating part of the tree of life. Fungal microbes have emerged as a particularly powerful system to better understand the causes and consequences of ploidy variation (Gerstein and Otto 2009). We first document cases in diverse fungal taxa where within-species ploidy variation has been identified, both in natural and in vitro systems. Second, we discuss the theoretical interplay between selection, mutation and drift in driving evolutionary ploidy change. Finally, we highlight experimental approaches that will help to fill the gaps in our understanding of ploidy evolution. 


\section{Within-species ploidy variation: Saccharomyces cerevisiae}

The budding yeast Saccharomyces cerevisiae has long been a model genetic organism and was the first eukaryote with a fully-sequenced genome (Goffeau et al. 1996). The utility of $S$. cerevisiae for empirical genetic testing follows in part from its ability to grow as a stable haploid or diploid in the laboratory (Sherman 1991; Liti 2015). Most laboratory strains cannot switch mating type, allowing for haploid cultures of a single mating type to be maintained. Like most fungi, S. cerevisiae is facultatively sexual (Nieuwenhuis and James 2016), though the frequency of outcrossing in $S$. cerevisiae is estimated to be just $2 \times 10^{-5}$ per asexual generation (Ruderfer et al. 2006). This low rate of sexual reproduction and flexibility in the reproductive system may allow for greater tolerance of ploidy variation.

Ploidy variation is found among isolates of $S$. cerevisiae from all of its known ecological niches. The first recorded polyploid wild isolates (i.e., those not directly associated with humans) were identified from Evolution Canyon in Israel (Ezov et al. 2006). Since then, several large-scale surveys of wild and domesticated strains have uncovered various degrees of ploidy variation from around the world (Table 1). Overall, $~ 75 \%$ of isolates are diploid. A lower frequency of diploids seems to be associated with alcohol production (with an overabundance of polyploids; Al Safadi et al. 2010; Gallone et al. 2016; Peter et al. 2018), clinical isolates (an increased number of haploids and polyploids; Zhu, Sherlock and Petrov 2016) and the relatively harsh environment of the wild Israeli strain set from Evolution Canyon (compared to forests where most other wild isolates originate; Ezov et al. 2006). In some cases ploidy variant strains form monophyletic groups (Ezov et al. 2006; Zhu, Sherlock and Petrov 2016; Peter et al. 2018), while in others cases they are phylogenetically dispersed (Duan et al. 2018). There appears to be considerable diversity in the prevalence of ploidy variation and its phylogenetic clustering, implying that ploidy evolution is highly context-specific.

\section{Ploidy variation is prevalent in many fungal species}

Although fewer studies characterize isolates from other fungal species, natural ploidy variation in fungi is not restricted to $S$. cerevisiae (Figure 1). Ploidy variation is observed within species isolated from ecological, clinical, and human-associated environments that span the fungal phylogeny. The peculiarities of fungal naming conventions are a potential complication worth mentioning. Until 2013, it was permissible for taxonomists to assign one name to the sexual (teleomorph) form and a different name (in an entirely different genus) to the asexual form (the anamorph; Hawksworth 2011). In other cases, different names were given to the same species 
when isolated from clinical or industrial contexts. As sexuality imposes a much stronger constraint on ploidy variation than asexuality, here we have used the anamorph names (Brandt and Lockhart 2012) but conducted literature searches using all name variants. We focus our discussion on species that grow as yeast, but note that ploidy variation within multicellular individuals in the form of multinucleate cells is also observed (e.g., in the filamentous fungi Ashbya gossypii (Anderson et al. 2015).

Consistent with the patterns observed in S. cerevisiae, ploidy variation has been seen in many diverse species that are opportunistic pathogens of humans. Both haploid and tetraploid clinical isolates have been found in the well-studied predominantly-diploid species Candida albicans, which is often found as a human commensal (Schulze and Sonnenborn 2009). In Cryptococcus neoformans, a saprophytic haploid species that can also become pathogenic, up to $\sim 20 \%$ of the population in the human lung is composed of large, polyploid "Titan" cells that are implicated in human virulence (Okagaki et al. 2010; Zaragoza et al. 2010). Until recently, Titan cells were only isolated from the hypoxic lung environment of humans or mice, and it was a challenge to identify the specific in vitro conditions that induced Titan cell formation. As summarized by Zhou and Ballou (2018), Titan cell formation (i.e., rapid polyploidization) in vitro was shown to be possible with growth in a low-nutrient medium and to be influenced by exposure to the bacterial cell wall component peptidoglycan Muramyl Dipeptide, as well as $\mathrm{pH}$, hypoxia and temperature (Dambuza et al. 2018; Hommel et al. 2018; Trevijano-Contador et al. 2018). The ability to form Titan cells under appropriate in vitro conditions has also now been observed in related species in the Cryptococcus neoformans/Cryptococcus gattii species complex (Trevijano-Contador et al. 2018; Dyląg, Colon-Reyes and Kozubowski 2020). Triploid strains have also been found in isolates of the diploid species Candida krusei, which is used industrially in chocolate production but has also emerged as a source of clinical infection (7 of 32 isolates were triploid, including isolates of clinical and industrial origin; Douglass et al. 2018).

Ploidy variation is not always observed. No diploid (or higher ploidy) isolates have been recovered from Candida glabrata, a haploid species (Carreté et al. 2018; Gabaldón and Fairhead 2019). It is likely that other studies have looked for but not found ploidy variation, but also likely that unidentified ploidy variation exists in strain collections from additional species. 
Fewer recorded examples of intraspecific ploidy variation are found in non-clinical species; whether this reflects a human bias in what we study (and where we study it), or a biological difference is unknown. Diploid, triploid and tetraploid isolates are found among geographically diverse isolates of the chytrid Batrachochytrium dendrobatidis (Bd; Farrer et al. 2013; Rosenblum et al. 2013). Geographically diverse diploid and triploid isolates have been observed in approximately equal numbers (42\% and $58 \%$ respectively) from Brettanomyces bruxellensis, a yeast species that is a contaminant of industrial fermentations (Borneman et al. 2014; Cibrario et al. 2019). Interestingly, the triploid isolates showed increased ethanol and sulphite tolerance, and the proportion of triploids has increased over time, which researchers suggest reflects adaptation to winemaker practices under artificial selection (Borneman et al. 2014; Cibrario et al. 2019). Rarer ploidy variants also exist: a single diploid isolate was identified among 57 diverse strains of the normally haploid Schizosaccharomyces pombe collected worldwide and deposited in stock centers (Jeffares et al. 2015).

Ploidy diversity has also been observed among different oomycetes species, a distinct group of eukaryotic microbes more closely related to brown algae than fungi. In Phytophthora infestans, the species that causes potato blight (Li et al. 2017), asexual lineages predominantly range from diploid to triploid (with a handful of rare tetraploids), with many non-euploid clones observed (Daggett, Knighton and Therrien 1995; Yoshida et al. 2013; Li et al. 2017; Knaus et al. 2020). In Phytophthora capsici, which causes disease in peppers, diploids, triploids, and a single tetraploid were found among 38 isolates within three different genetic groups collected from Sweet pepper, Chili pepper and Tomato plants from fields in Taiwan (Barchenger et al. 2017) and among isolates from research field populations (Carlson et al. 2017). Although there was no direct correlation between virulence and ploidy, many of the triploid isolates were less virulent than the historical diploid isolates (Barchenger et al. 2017). Ploidy variation linked to geography was found in 89 isolates of the Taro Leaf Blight species Phytophthora colocasiae: Hawaii isolates were primarily diploid; China isolates were primarily triploid; Vietnam isolates were diploid and triploid; Nepal isolates were predominantly triploid, tetraploid, and higher (Shrestha et al. 2017). We note that similar to the fungal taxa, studies of oomycete species are also biased towards those that are plant pathogens.

\section{Potential limitations in assessing variation}

While ploidy variation has been documented in many organisms, it is important to address some practical limitations in detecting such variation. As we describe below, ploidy is not 
necessarily a "hard-wired" trait, and can change very rapidly under laboratory conditions. For example, haploid strains of Candida albicans that are isolated in vitro tend to rapidly diploidize under laboratory culture, and even during shipping from one lab to another (Hickman et al. 2013). Many studies use flow cytometry to measure ploidy, which requires adding a DNA stain to a large population of cells. This often requires culturing samples to single colonies from frozen stocks before the fixation of cells for analysis, such that multiple passages take place between isolation and fixation. As a consequence, there is some opportunity for ploidy variation to arise among colonies before analysis. On the other hand, population-level flow cytometry profiles (where a culture is not reduced to single colonies) may hide rare ploidy variants segregating within the population. Even when multiple ploidy states are clearly present within a sample, we currently lack robust high-throughput methods for quantifying this variation and reporting statistical uncertainty and confidence limits on inferred genome sizes.

Furthermore, flow cytometry traces are often imperfect, and it is not standard practice to make them publicly available. Other protocols that infer ploidy by examining allelic ratios (e.g., RADseq) suffer from many of the same empirical limitations. Until technological advances such as culture-free single-cell genomics are commonly available to catalogue natural variation, we caution that what we have described may underestimate the true incidence of ploidy variation in the wild.

\section{Why is there ploidy diversity?}

Given that inter- and intraspecific ploidy variation is phylogenetically widespread, the obvious question is: why? Why isn't there a single best ploidy level? This is not a new question to ask, nor is it specific to the microbial taxa that we have discussed here. There is a rich history in theoretical and empirical literature, particularly in plants and algae, that seeks to understand why ploidy variation occurs.

As described above, there are many examples of ploidy variants sampled from natural populations. However, many studies that characterize natural isolates are done somewhat haphazardly, based on sample availability and often relying on stock centers and repositories. Experimental evolution studies provide another way to shed light on the potential role of the environment in shaping ploidy diversity. In these experiments, the researcher has precise control over the environmental conditions that a population is exposed to in the lab and can evolve many replicate populations from the same ancestral culture, thereby partially 
disentangling the roles that environment and genetic background play in the evolution of ploidy transitions. Such in vitro evolution experiments have documented both ploidy increases and decreases in the lab (Figure 1). Unregulated ploidy doubling can happen in a single step by endoreduplication or multiple steps by a mating-type switching event (which is usually prevented by genetic manipulation) followed by mating (Harari et al. 2018). Ploidy reduction in fungi seems to occur through aneuploid intermediates, yet euploid or near-euploid shifts can happen within a small number of cell divisions, suggestive of a concerted process (e.g., in $S$. cerevisiae: Gerstein et al. 2008; Candida albicans: Forche et al. 2009, C. neoformans: Gerstein et al. 2015). It remains unknown whether ploidy reduction mechanisms in diverse taxa (e.g., the ascomycete S. cerevisiae and the basidiomycete Cryptococcus neoformans) are mechanistically similar.

An early evolution experiment (at the time referred to as "adaptation by recycling") in an industrial yeast species maintained one strain (Candida shehatae, the anamorph) for 72 transfers and a second strain (Pichia stipitis, the teleomorph) for 12 transfers in wood hydrolysate solutions (Parekh, Yu and Wayman 1986). Both evolved populations were later found to have doubled their ploidy relative to the ancestral strain (Talbot and Wayman 1989), leaving the researchers to conclude that the increased efficiency in xylose fermentation observed in the evolved strains was at least partially due to their doubled ploidy level.

More recent experiments also find rapid ploidy change, within 50-1000 generations of laboratory adaptation. Perhaps surprisingly, these ploidy changes often occur without noticeable fitness improvements attributable to the shift in ploidy per se in the evolutionary environment (i.e., ploidy change independent of other new mutations or beneficial chromosomal aneuploidies). For example, in an experiment with Phytophthora infestans, a diploid clone derived from a triploid isolate was identified after treatment with metalaxyl, yet there was no apparent change in fitness between the evolved diploid and the triploid ancestor (Li et al. 2017).

Rather than a close link between ploidy and fitness changes, the overarching pattern of many experiments is evolution back towards the historical or baseline ploidy state. Many batch culture experiments (also referred to as experimental evolution or adaptive laboratory evolution) have now shown genomic convergence towards the base genome size in aberrantploidy replicate populations, as well as high stability of base-ploidy populations in $S$. cerevisiae 
(base diploid; Mable and Otto 2001; Gerstein, McBride and Otto 2008; Oud et al. 2013; Selmecki et al. 2015; Voordeckers et al. 2015; Lu, Swamy and Leu 2016; Kosheleva and Desai 2018), Candida albicans (base diploid; Hickman et al. 2015; Gerstein et al. 2017), Aspergillus nidulans (base haploid; Schoustra et al. 2007), and Cryptococcus neoformans (base haploid; Gerstein et al. 2015). Note that these experiments include very distantly related fungal species, separated by hundreds of millions of years of evolution (Willis et al. 2018).

To catalogue the generality of ploidy change towards the base genome during in vitro experiments, we compiled the data from 22 manuscripts (Supporting Table 1, live document: https://www.microstatslab.ca/invitro.html). We included all manuscripts we found that evolved at least two replicate populations from the same ancestor. These experiments were performed using various experimental methods (batch culture transfers, chemostats, turbidostats, mycelial transfer) and compared evolutionary patterns from different starting ploidy states, genetic backgrounds, and environments (Figure 2). The overwhelming pattern-that populations starting at a non-base ploidy level evolve towards their species' baseline ploidy-led us to previously coin the term "ploidy drive" to describe this phenomenon (Gerstein et al. 2017). Although it is tempting to draw additional conclusions from the patterns here, we will refrain from doing so, given the many factors (described below) that can influence the rate or frequency of change. For example, we note that these experiments were conducted over a range of evolutionary timescales, from a small number of transfers up to 2000 generations, with ploidy generally assessed only at the terminal time point. A lack of observed ploidy change in some, but not all, cases may therefore be attributable to a limited timescale. For example, the majority of initially-haploid isolates that remained haploid in these experiments were sampled after less than 300 generations.

The specifics of the environment influence the frequency of genome size change in multiple species, as demonstrated by studies that conducted replicate experiments from the same strain background in different stressful environments (Table S1; Bennett and Johnson 2003; Seervai et al. 2013; Hong and Gresham 2014; Gerstein et al. 2015, 2017; Gorter et al. 2017; Turanlı-Yıldız et al. 2017; Harari et al. 2018). Variation in the frequency of change has also been observed in different levels of the same stress (Turanlı-Yıldız et al. 2017; Harari et al. 2018) and in different evolution regimes when stress was added abruptly or gradually (Gorter et al. 2017). 
Although fewer studies have directly examined it, strain background (i.e., ancestral genotype) is also implicated in influencing the proportion of replicates that change in ploidy (Filteau et al. 2015; Gerstein et al. 2017; Gerstein and Berman 2020). Through laboratory experiments on targeted mutants and knockout strains, several genes have been identified that influence the rate of genome instability and ploidy change (e.g., genes involved in DNA replication and repair, the mitotic apparatus, and cell cycle control, reviewed in Todd 2017). Whether the strain background differences can be attributed to "natural" standing polymorphism in such genes remains to be determined. It's also very likely that hybridization will influence the spread of ploidy variants. This can be seen in Figure 2 and Table S1: an S. cerevisiae experiment where $50 \%$ of the isolates changed ploidy was initiated from a hybrid diploid, and those replicates became haploid (Kosheleva and Desai 2018).

A variant of batch culture has recently been developed that can also shed light on how ploidy variants arise and spread through populations. In "lineage-tracking" experiments, the relative frequencies of $\sim 500,000$ barcoded lineages are tracked simultaneously during batch culture growth, and lineages that increase in frequency (i.e., carry beneficial alleles) can be isolated and examined (Levy et al. 2015). The first major experiment that mapped genotype to phenotype using this framework examined 4800 clones of initially-haploid $S$. cerevisiae after 88 generations of evolution in glucose limiting conditions. A high number of clones became diploid ( $46 \%$ of cases where ploidy could be identified, based on their Table S1); the majority of characterized diploid clones were classified as adaptive, and many carried no other identified mutations (Venkataram et al. 2016). These results are consistent with the idea that diploidy (the base ploidy of $S$. cerevisiae) can have an immediate evolutionary advantage over haploidy in some environments. Still, the reasons for this advantage remain unclear.

\section{The population genetics of ploidy change}

The preceding observations tell us that the ploidy level of fungal species is not static; ploidy variability is observed among natural isolates, and ploidy can sometimes change rapidly during experiments, depending on the environment and genetic context. Understanding why and how ploidy evolution occurs will require more information about the population genetics of ploidy change.

Two different processes must occur for ploidy variants to rise to high enough frequency to be readily sampled, either from natural isolates or in lab populations. First, the ploidy variant must 
appear in the population. Second, it must rise in frequency, either because it is directly beneficial and favoured by selection, or indirectly, because it opens up a new avenue for subsequent adaptation. Thus in examining the evolutionary trajectory of ploidy-variant cells, we are actually talking about two distinct rates: the spontaneous rate that unregulated ploidy changes arise in populations (driven by mutation) and the rate that ploidy variants sweep through populations (driven by short- and long-term selection). Genetic drift can also affect the frequency of ploidy variants, particularly when the effective population size is small.

\section{The mutation rate of ploidy}

While the distinction between the rate of mutation and the effect of a mutant allele on fitness may seem obvious, these concepts are sometimes confounded when describing genetic change in experimentally evolving populations. The term "rate" could refer to the number of generations it takes for a new allele to spread to fixation or the number of populations in which fixation occurs in a given time frame. Either of these rates will depend partly on the strength and effectiveness of selection, and partly on the underlying mutation rate for the variants of interest. For example, suppose the frequency of populations in which one ploidy type replaces another is found to differ between environments or genetic backgrounds; this could mean that the alternative conditions confer different rates of ploidy mutation, different selective regimes, or both. To fully understand ploidy evolution, we will need to distinguish between the effects of mutation, selection, and drift (Box 1), and recognize circumstances where these processes have confounding effects on our observations.

Experimental evolution through batch culture (or chemostats and turbidostats) is powerful because it provides an experimental framework for the researcher to precisely control the input (the strain, the environment), with the evolutionary outcome determined by the natural processes of mutation, selection and drift (Cooper 2018). However, these experiments do not directly address the rate of ploidy mutation per se, meaning the number of spontaneous ploidy changes per genome per generation, regardless of whether such changes are beneficial, deleterious, or neutral.

\section{Fluctuation assays}

Microbiologists have generally employed two different plate-based experimental methods to measure mutation rates (Figure 3). Fluctuation assays were first developed by Luria and Delbrück (1943) to examine properties of spontaneous mutation rates in bacteria. In this 
framework, a culture of microbes is grown up from a small population to a large population in a non-selective medium in many replicate cultures, and some cells in the population acquire mutations. The population is then plated onto both non-selective medium and medium where only (typically rare) cells that carry mutations that confer the required phenotype will be able to form a colony. By comparing the number of colonies on the two plates (incorporating other parameters such as the initial and final number of cells and the number of parallel cultures), the mutation rate to the selectable phenotype can be inferred (Foster 2007, Pope et al. 2008, Lang 2018).

In fungal microbes, ploidy variant cells have been identified in fluctuation assays that directly selected for genome instability, and as an unexpected by-product of selecting for other mutations. Bennett and Johnson (2003) first documented the parasexual cycle in Candida albicans through a fluctuation assay. They constructed a counter-selectable tetraploid strain that was heterozygous for URA3 and GAL1. By plating on medium containing both 5Fluoroorotic acid (5-FOA; on which strains wildtype for URA3 are unable to grow) and 2deoxygalactose (2-DOG; on which strains wildtype for GAL1 are unable to grow), they estimated that growth at $37^{\circ} \mathrm{C}$ in "pre-spo" medium induced chromosome loss $\sim 10$ times faster than other tested conditions (Bennett and Johnson 2003; Filteau et al. 2015; Forche et al. 2018). A diploid strain heterozygous for GAL1 was similarly used to examine genome instability of Candida albicans following passage through a mouse (isolated after 1-5 days from tongue homogenates). An examination of 541 colony isolates from non-selective plates and 360 colony isolates from 2-DOG plates identified 11 haploid isolates and seven tetraploid isolates (Bennett and Johnson 2003; Filteau et al. 2015; Forche et al. 2018). An experiment in $S$. cerevisiae compared the compensatory mutation rate to las 17-41 (a yeast allele that is homologous to the human gene WASP that causes Wiskott-Aldrich syndrome). The assay was done in two haploid las 17-41 genetic backgrounds, each grown on glucose and galactose. Similar to experimental evolution results, both genetic background and the growth environment influenced the rate that diploid colonies were isolated in these fluctuation assays (Bennett and Johnson 2003; Filteau et al. 2015; Forche et al. 2018).

In these experiments, precise mutation rates weren't estimated. It's important to bear in mind that by purposefully selecting on medium and requiring the loss of an allele through recombination, loss-of-heterozygosity, or aneuploidy, this approach may produce a biased view of the mutation rate since it selects for genome instability. In population genetics terms, 
although fluctuation assays can be done in a manner that takes into account the stochasticity of the evolution process (specifically, the timing of when mutations arise within a population), thus far the fluctuation experiments that document ploidy variation have not done so. These experiments can undoubtedly be useful as a coarse method to examine how the environment influences differences in the beneficial mutation rate. Still, unless designed purposefully, they will not precisely measure the mutation rate of ploidy.

Harari et al. (2018) combined batch culturing, fluctuation tests, flow cytometry, PCR of the MAT locus, and population modelling to estimate rates of diploidization in haploid $S$. cerevisiae, with a focus on stressful environments, particularly $5 \%$ ethanol. Their estimate of the spontaneous endoreduplication rate of MATa cells in this environment is about $5 \times 10^{-5}$, with a much lower rate observed in non-stressful conditions. While labour-intensive, this is a welcome effort to quantify rates of ploidy change under alternative conditions.

\section{Mutation accumulation}

A second useful procedure for measuring mutation rates is mutation accumulation (MA). In these experiments, many independent lineages, which are generally derived from a common ancestor, are subjected to repeated population bottlenecks over many generations. These bottlenecks cause the MA lineages to behave like very small populations in terms of their genetics, i.e., they have a small "effective population size", $N_{e}$. Within each MA line, $N_{e}$ is too small for selection to act effectively on most new mutations, allowing them to become fixed and accumulate as though they were selectively neutral. The mutations in each line can then be determined by sequencing. Dividing by the number of generations of MA allows the rate of mutation per genome (or site) per generation to be calculated. As an alternative to sequencing, ploidy changes can be scored by using flow cytometry or other measures of genome size. In essence, MA experiments measure the pace of evolution via mutation alone, rather than the rate of adaptation, which is influenced by both the mutation rate and the efficacy of selection.

MA experiments also have limitations. Mutations with very large fitness effects will still be subject to effective selection in small populations, such that strongly deleterious mutations will accumulate more slowly than their true mutation rate, and strongly beneficial mutations will accumulate more rapidly than their true mutation rate. Whether a mutation will be subject to selection depends on its fitness effect, $s$, and the effective population size, $N_{e}$. In theory, 
mutations in a haploid population with $|s|<<1 / N_{e}$ will have virtually the same probability of becoming fixed as neutral alleles (where $s=0$ ) and are described as effectively neutral. In microbes, MA experiments are typically conducted by using a single random colony to streak out new single colonies, which are presumed to grow from individual cells, resulting in lineages that have undergone periodic single-cell bottlenecks. In this scenario, the effective population size can be approximated as the harmonic mean population size (Box 1). For example, assuming exponential growth, 16 generations per day, and daily single-cell bottlenecks (similar to some yeast MA experiments) $N_{e} \cong 8.5$ (Figure 3), and we would expect mutations with absolute selective effects much less than about $12 \%$ to be effectively neutral. While the distribution of fitness effects of new mutations remains poorly understood, it appears likely that most mutations have effects that are weaker than this threshold, particularly in the heterozygous state (Eyre-Walker and Keightley 2007; Böndel et al. 2019). As we will discuss below, there is currently little evidence that the fitness effects of ploidy shifts are of this magnitude, so MA should be a viable way to estimate the spontaneous rate of ploidy mutations.

An additional caveat to estimating mutation rates from MA experiments is the assumption that back mutations do not occur. For most types of mutations, this is not a significant problem because the probability of back mutation during the experiment is negligible. Whether or not this is also the case for ploidy changes is unknown, and could depend on the initial ploidy state of the MA lines (Figure 2). For example, in S. cerevisiae many instances of haploid-to-diploid mutations have been observed, including in some MA experiments (discussed below), but the reverse change appears to be less frequent. If this reflects an actual mutational bias towards diploidy in this system we might expect mutations away from diploidy to have a high chance of reversion, making them more challenging to detect in an MA experiment. In general, back mutation has the potential to create a downward bias in mutation rate estimates whenever the rates of forward and back mutation are of similar orders of magnitude.

In an MA experiment with S. cerevisiae (Lynch et al. 2008), all four lines analyzed were found to have switched from haploidy to diploidy by the end of the $\sim 4800$-generation experiment, indicating that ploidy increases occurred at a rate of at least $2 \times 10^{-4}$ per genome per generation ( $95 \%$ confidence interval: $5.7 \times 10^{-5}$ to $\left.5.3 \times 10^{-4}\right)$. A later study of $>100$ haploid MA lines found no ploidy changes after $\sim 1600$ generations (Sharp et al. 2018), implying a rate of ploidy shifts no higher than $2.3 \times 10^{-5}$ per genome per generation (the upper $95 \%$ confidence 
limit for these data). Serero et al. (2014) studied 44 MA lines from ten genetic backgrounds (mutator genotypes and wild-type) and found one switch from haploidy to diploidy in a mutator strain (rad27 $)$, for an overall rate of $1.1 \times 10^{-5}$ across the genotypes (95\% Cl: $3 \times 10^{-7}$ to $6.2 \mathrm{x}$ $\left.10^{-5}\right)$. These conflicting results could reflect a difference in the rate of ploidy changes between genetic backgrounds or environments, which are factors known to influence the rate of mutations in various eukaryotic organisms (e.g., Yauk et al. 2008; Wang and Agrawal 2012; Schrider et al. 2013; Harris 2015; Sharp and Agrawal 2016; Berger et al. 2017; Chain et al. 2018; Harari et al. 2018; Saxena et al. 2018; Liu and Zhang 2019).

MA experiments using diploid S. cerevisiae have not observed any ploidy changes (Sharp et al. 2018, Zhu et al. 2014), indicating a rate of ploidy change no higher than $7.7 \times 10^{-6}$ in diploids (the upper $95 \%$ confidence limit from pooling these two studies). A study of tetraploid S. cerevisiae found that two out of ten MA lines transitioned to diploidy within 566 generations, suggesting a rate of at least $3.5 \times 10^{-4}\left(95 \% \mathrm{Cl}: 4.3 \times 10^{-5}\right.$ to $1.3 \times 10^{-3}$; Gerstein et al. 2006), similar to some observed rates of change from haploidy to diploidy. When maintained in batch culture, all five initially-tetraploid populations from the same genetic background became diploid over the same timeframe, suggesting that selection favoured diploids in this case (Gerstein et al. 2006). MA experiments conducted with haploids of the fission yeast Schizosaccharomyces pombe, in which haploidy is the baseline state, revealed no instances of ploidy shifts (Farlow et al. 2015, Behringer and Hall 2016), consistent with a rate of diploidization no higher than $1.2 \times 10^{-5}$ in this species (the upper $95 \%$ confidence limit from pooling these two studies). Therefore, the available evidence from two distantly-related yeast species suggests that spontaneous transitions away from the baseline ploidy state are rare.

Additional MA experiments would be valuable for obtaining precise estimates of spontaneous rates of ploidy change. Still, the available data from $S$. cerevisiae suggests that ploidy may be subject to mutational bias, where mutations towards baseline ploidy are at least an order of magnitude more likely than mutations away from baseline ploidy. Under these conditions, we would expect the population to be predominantly diploid at equilibrium in the absence of any fitness difference between ploidy states (see Box 1).

\section{The fixation of ploidy variants}

While ploidy variant cells may often arise because of mutation, they will not necessarily reach sufficient frequency to be sampled in laboratory experiments. Classic population genetics 
theory on "origin-fixation dynamics" (Kimura 1962) predicts that the probability that a new mutation will ultimately become fixed rather than lost depends on its initial frequency $p_{0}$, the selection coefficient favouring or disfavoring the mutant type $(s)$, and the effective population size $\left(N_{e}\right)$. The dominance coefficient, $h$, is also relevant for typical mutations in diploid genomes, where $h$ modifies the selection coefficient when the mutant allele is not homozygous. For a "cellular variant" like ploidy, the fixation probability in an asexual population can be considered analogous to that of a new mutation in a haploid population, since the initial frequency of a single variant will be $1 / N$, where $N$ is the population size, and each cell can have only one possible ploidy state, analogous to an allele. (In this case a dominance coefficient would not apply, but note that sex between alternative ploidy states could generate "intermediate" states, complicating this analogy). The probability of fixation for a non-neutral variant in a haploid population is approximately $\operatorname{Pr}(\mathrm{fix}) \cong\left(1-\mathrm{e}^{-2 \mathrm{Ne} s \mathrm{p} 0}\right) /\left(1-\mathrm{e}^{-2} \mathrm{Nes}\right)$ (Moran 1962; Kimura and Crow 1964; Otto and Whitlock 2006). The initial frequency of a new mutation is typically assumed to be $p_{0}=1 /(c N)$, where $N$ is the census population size and $c$ is the number of genome copies per cell, because multiple mutations to the same allele are unlikely to occur during the period of time in which the mutant allele is segregating. However, this assumption may not hold for ploidy variants, which may have a much higher mutation rate than individual nucleotide sites.

If $N_{e} s$ is large, a variant starting as a single copy (frequency $1 / M$ ) has $\operatorname{Pr}($ fix $) \cong 2 s N_{e} / N$. This result illustrates that even strongly-beneficial variants have an appreciable probability of becoming lost by chance. If the fitness effect of a variant is weak and the effective population size is relatively small, such that $N_{e}|s|<<1$, non-neutral variants have essentially the same probability of fixation as neutral variants: $1 / \mathrm{N}$. In other words, deleterious or neutral mutations can become fixed when $N_{e}|s|$ is small (as in mutation accumulation experiments). Note that fluctuations in population size, such as those experienced by many laboratory populations maintained by serial transfers, will also affect the effective population size and the probability of fixation (Otto and Whitlock 1997, Patwa and Wahl 2008).

It is also important to recognize that deleterious variants can reach a considerable frequency even if they do not ultimately become fixed. In a stable population where $N_{e}|S|$ is not too small, the equilibrium frequency of the deleterious ploidy type, $q^{*}$, is approximately $\mu / s_{d e l}$, where $\mu$ is the mutation rate to the ploidy variant and $s_{d e l}$ is the deleterious effect. This phenomenon, called mutation-selection balance, may be particularly relevant for ploidy changes, where there 
is evidence that the mutation rate is not too low. For example, if $\mu=2 \times 10^{-4}$, as observed by Lynch et al. (2008) for transitions from haploidy to diploidy in S. cerevisiae, we would expect $20 \%$ of the population to be diploid at equilibrium with $s_{d e l}=0.1 \%$.

In summary, detecting fixed or segregating ploidy variation in experimental populations is insufficient to infer the effects of ploidy variation on fitness in a given environment, particularly in demographically-complex populations. Ideally, we would obtain unbiased estimates of both the mutation rate (see above) and the direct fitness consequences of ploidy variation. In the next section, we will describe some of the evidence and challenges associated with measures of the relative fitness of alternative ploidy states.

\section{Short- and long-term consequences of ploidy change}

Changes in ploidy can have immediate phenotypic effects, but we also expect such changes in genome architecture to have longer-term effects by altering the nature of mutation or selection throughout the genome. The immediate consequence of a change in ploidy that is most relevant from a population genetics perspective is the fitness of ploidy-variant cells relative to that of their contemporaries. In the simplified scenario where there is no genetic variation in the population apart from the ploidy variant, any difference in fitness can be attributed to ploidy itself. In Box 2, we examine the dynamics of this scenario using simulations. In reality, a ploidy variant will arise within a particular genetic background, such that its fitness effect could either be direct or a consequence of interactions with alleles on that genetic background, analogous to an epistatic interaction between two alleles. Other genetic variants could also co-occur with ploidy if mutations arise in the novel background simultaneously or shortly after the ploidy shift. Such interaction effects may be more relevant to the fate of ploidy variants than other genetic changes due to the high mutation rate of ploidy, which will increase the chances that a ploidy variant will co-occur with other mutations.

In addition to these initial genetic combinations, the frequency trajectory of a segregating ploidy variant will depend on what other mutations subsequently arise within the population on all genetic backgrounds. In an asexual population, multiple new genotypes may compete for fixation, a phenomenon known as clonal interference (Muller 1932). In the context of experimental evolution, the potential for multiple (and potentially non-transitive) fitness interactions (e.g., Buskirk, Rokes and Lang 2019), means that the fitness of ploidy variants 
should be compared to the other cells in the population at the time the variant is first present (ideally, the isogenic non-variant) rather than against the ancestral genotype.

\section{Immediate phenotypic changes}

Changes in ploidy can have immediate consequences, which may or may not be beneficial. Cell size and shape is the most obvious consequence that accompanies a ploidy change in fungal microbes. Although the distribution of cell sizes are overlapping between ploidy levels (Jorgensen et al. 2007, Zadrag-Tecza et al. 2018), cell and nuclear volume increase approximately proportionally as ploidy increases in S. cerevisiae (Mundkur 1953; Mortimer 1958; Jorgensen et al. 2007; Gerstein and Otto 2011; Zadrag-Tecza et al. 2018), Candida albicans (Hickman et al. 2013), and Schizosaccharomyces pombe (Neumann and Nurse 2007). Size increases lead to an increased requirement for biosynthetic capacity, and ATP content and glucose uptake rate both generally increase with an increasing number of genome copies in S. cerevisiae (though interestingly, not necessarily in a proportional fashion, ZadragTecza et al. 2018). A non-linear scaling of linear and two-dimensional structures, such as membranes, also accompanies cell volume doubling (Storchova 2014). These size differences do not seem to disrupt the processes of DNA replication or translation: for the majority of genes ploidy did not affect relative mRNA (1n, 2n, 3n, 4n: Galitski et al. 1999; $1 n$ vs. $4 n$ : Wu et al. 2010) or protein levels ( $1 n$ vs. $2 n$ : de Godoy et al. 2008; $2 n$ vs. $4 n$ : Storchova 2014), nor on the length of the different phases of the cell cycle (1n vs $2 n$ : Brewer et al. 1984; $2 n$ vs. $4 n$ : Storchová et al. 2006).

Increasing ploidy also changes the cell shape from spherical towards elliptical (i.e., higher eccentricity; Gerstein and Otto 2011; Zadrag-Tecza et al. 2018). Even with the same shape, as the cell volume increases, the surface area to volume ratio (SA:V) decreases, hence higherploidy cells have lower SA:V. Some biological processes such as membrane synthesis are known to scale with SA:V (Schmoller and Skotheim 2015), and ecological factors such as nutrient limitation or toxin exposure could potentially provide ploidy variants with an immediate advantage in specific environments due to their SA:V.

Experiments that look for a correlation between ploidy and fitness have produced mixed results. Mable (2001) found that $S$. cerevisiae ploidy did not predict fitness well under nutrient limitation. Tetraploids consistently grew slower than haploids or diploids, and although haploids grew faster than diploids under nutrient-rich conditions, there was no difference in competitive 
fitness between them. Zheng et al. (2017) examined an isogenic ploidy series of $S$. cerevisiae in YPD and vanillin, a major phenolic aldehyde compound that inhibits bioethanol production from lignocellulosic biomass. They found that in YPD media haploids and diploids proliferated more rapidly and formed more biomass than triploid and tetraploid strains. In the presence of vanillin, the triploid strain initially had the shortest lag phase and formed the most mass after 3 days, though after 5 days haploid and diploid strains formed more biomass and again had higher growth rates compared to triploids and tetraploids. Other studies have occasionally found a benefit to one ploidy level over another in specific environments but not others. For example, Mable and Otto (2001) found that haploids were more sensitive to EMS treatment at multiple levels than either diploids or tetraploids (with no advantage to tetraploids over diploids; tetraploids actually became diploid with repeated exposure). In a study that examined resistance to cellular inactivation by X-rays, a ploidy series of $S$. cerevisiae strains from haploid to hexaploid determined that diploids were the most radioresistant (Mortimer 1958). Storchová et al. (2006) similarly found diploids were less sensitive than tetraploids to agents that induce double-strand breaks or are detrimental to microtubules, while no difference was observed under osmotic stress, oxidative damage, or UV light. Peter et al. (2018) collected hundreds of $S$. cerevisiae isolates from various environments, most of which were diploid, and found that the diploid isolates showed the fastest mitotic growth, on average, across environments. However, these isolates have many genetic differences apart from ploidy, making it difficult to discern the role of ploidy per se. The most comprehensive study of haploid and auto-diploid $S$. cerevisiae and $S$. paradoxus natural isolates grown in 33 different environments did not establish a decisive advantage for one ploidy type (Zörgö et al. 2013), including in environments such as nutrient limitation or in the presence of toxic drugs where a ploidy advantage due to differences in cell size or shape has been predicted. In the data from Zörgö et al. (2013), interaction effects between the environment, ploidy and genetic background are widespread, underscoring the need for caution when ascribing results from a single genetic background in a specific environment to a generalizable benefit of a given ploidy level.

Caution is also needed in interpreting the observations of "ploidy drive" we previously described, where populations tend to evolve towards the baseline ploidy state. It is tempting to conclude that cells of the baseline ploidy must have an immediate fitness advantage in order to take over the population, but other alleles with ploidy-specific effects could also be responsible. Approximately $1 \%$ of genes that are essential for survival or are temperature sensitive in 
tetraploid S. cerevisiae are not essential in haploids or diploids (Storchová et al. 2006). A set of beneficial mutations (18 point mutations, one large duplication and one large deletion) acquired in haploid lines of $S$. cerevisiae in response to the drug nystatin tended to provide a higher benefit to haploid cells than isogenic homozygous diploid cells (Gerstein 2013). Similarly, chromosome XIII aneuploidy in S. cerevisiae, which provides a fitness benefit in raffinose medium, was more beneficial in the tetraploid background on which it arose than on a diploid background (Selmecki et al. 2015). These results suggest that mutations with ploidyspecific beneficial effects could either favour a baseline or non-baseline ploidy state. Accordingly, in some cases where ploidy drive has been observed there may be an immediate fitness benefit to diploidy (e.g., Voordeckers et al. 2015; Venkataram et al. 2016), while other cases indicate this is not a generalizable result (Gerstein and Otto 2011; Hong and Gresham 2014).

It is also important to examine competitive fitness, rather than growth rate or other fitness proxies, when trying to understand the evolutionary dynamics of ploidy change. Isolating $S$. cerevisiae ploidy-variant cells from within an experimental evolution study failed to identify a competitive advantage to diploid cells over haploids at the time point where diploids were first observed in appreciable frequency in either YPD or salt (two environments where ploidy drive to diploidy was observed; Gerstein and Otto 2009; Gerstein et al. 2011). Diploid S. cerevisiae lines that arose within a haploid mutation accumulation study similarly failed to exhibit a competitive benefit over lines that remained haploid, and no competitive difference was found between parental haploids and matched diploids (Dickinson 2008). Other experimental evolution studies in $C$. albicans that observed a rapid change in genome size also failed to establish a correlation between fitness and ploidy (Gerstein et al. 2017; Gerstein and Berman 2020). Unlike 'typical' mutations, ploidy has the potential to both alter phenotypes directly and modify longer-term genomic patterns. Direct effects appear inadequate to explain the variation in ploidy we observe in many experimental and natural systems.

\section{Long term genotypic consequences}

Once ploidy-variant cells are present in a population, longer-term evolutionary dynamics may be required to understand why a given ploidy level sweeps to fixation, particularly in cases where there is no obvious immediate benefit to the ploidy change. The "masking" of recessive deleterious mutations in the heterozygous state is often cited as a possible benefit of diploidy (or higher ploidy levels) over haploidy. Recessive deleterious mutations are common (reviewed 
in Charlesworth and Willis 2009), and so the effect of a typical mutation on haploids (s), will be greater than the effect on heterozygous diploids $(h s)$, as long as the mutant allele is not dominant $(h<1$, where $s$ is the selection coefficient, $h$ is the dominance coefficient, and $s$ is assumed to be the same in haploids and diploids). However, in terms of the long-term effect of deleterious mutations on fitness, the masking effect is temporary.

One way to consider the long-term effect of deleterious mutations on fitness is through mutation load theory (Haldane 1937). Mutation load is the extent to which the mean fitness of a population is reduced because of deleterious mutations, which appear continuously and are removed by selection. A classic theoretical result is that mutation load depends only on the mutation rate, and not on the effects of mutations on fitness. The reason is that mutations with mild effects will reach a relatively high equilibrium frequency because they are only weakly disfavored by selection, whereas mutations with severe effects will be held at low frequency by selection. The net result is that the effect size and frequency of mutations counterbalance one another, and mutation load depends only on the rate that deleterious mutations arise. The transient advantage to increased ploidy occurs because higher ploidy levels have more capacity for masking recessive deleterious alleles, and the frequency of such alleles won't immediately reflect the new expectation under mutation-selection balance, which will be higher due to the masking effect (see Otto and Whitton 2000 for discussion of this phenomenon in polyploids). In addition, higher ploidy states are expected to incur more mutations per genome (see below), increasing mutation load, but this consequence will manifest gradually as the population approaches the new state of mutation-selection balance.

How significant do we expect the temporary masking advantage to be? The fitness benefit of diploidy over haploidy will be greater when deleterious alleles have stronger effects, when deleterious alleles are more recessive, and when the genome-wide mutation rate is higher (including in mutator strains; see Tracy et al. 2020). In S. cerevisiae, knockout mutations appear to be moderately deleterious and partially-recessive, with a mean dominance $(h)$ of 0.2 , a mean deleterious effect $(s)$ of 0.045 , and a negative relationship between $h$ and $s$ (Agrawal and Whitlock 2011). In this organism, the masking benefit of diploidy is likely to be limited by the low mutation rate; based on results from Sharp et al. (2018), only about 0.002 nonsynonymous point mutations occur per diploid genome per generation in a lab strain. All else being equal, we therefore predict a negligible benefit to masking in yeast. Eventually, mutation-selection equilibrium will be restored, and the mutation load of all ploidy states will 
reflect their respective genome-wide deleterious mutation rates. As discussed below, higher ploidy levels will likely incur more mutations per genome per generation because of their larger genome size, such that haploids are expected to have the lowest mutation load. In multicellular organisms, the masking benefit of diploidy will apply to mutations that occur in somatic cells, which consistently show higher mutation rates than germline cells; diploidy may therefore help to limit rates of cancer and senescence (Orr 1995; Lynch 2010). Masking will also apply to recessive beneficial mutations, which could slow the rate of adaptation in diploids relative to haploids (see below).

Just as a transition from haploidy to diploidy could confer a short-term advantage by temporarily alleviating mutation load, we would expect a shift from diploidy to haploidy to expose any preexisting recessive deleterious alleles, representing a short-term disadvantage. This could lead to evolutionary stability of the diploid state, regardless of its long-term effects (Crow and Kimura 1965). However, recessive deleterious alleles can become homozygous in diploid populations through spontaneous loss-of-heterozygosity events or through sexual reproduction. Sex can promote the "purging" of deleterious variation, especially if it involves selfing, where gametes from the same parent combine. To the extent that genes are expressed during the haploid phase of a sexual life cycle, sex may help expose recessive deleterious alleles without involving a wholesale transition to haploidy (Immler and Otto 2018).

Sexual reproduction has additional long-term benefits. Theoretical studies predict that, by creating new allele combinations, sex and recombination allow selection to act more efficiently against deleterious alleles and in favour of beneficial alleles (Hill and Robertson 1966; Otto and Barton 1997; Keightley and Otto 2006; Sharp and Otto 2016). These predictions have been supported by laboratory experiments, particularly with budding yeast (Goddard, Godfray and Burt 2005; Gray and Goddard 2012; McDonald, Rice and Desai 2016). Sexual reproduction requires regulated transitions between ploidy states, which could be disrupted by unregulated ploidy changes. For example, in the absence of mating-type switching, diploidization in S. cerevisiae will result in cells with homozygous mating types that are incapable of sporulation. These cells may mate with haploids to form triploids, which produce highly-aneuploid progeny upon meiosis (St Charles, Hamilton and Petes 2010). In general, sporulation in polyploid budding yeast and fission yeast is often inefficient and results in aneuploid spores with poor viability (Storchova 2014). Therefore, spontaneous ploidy mutants are unlikely to persist except as asexual lineages, forgoing the long-term advantages of sex. 


\section{The rate and spectrum of mutations}

Classic models of ploidy evolution assume that the rate of deleterious mutation scales linearly with genome size (e.g., Crow and Kimura 1965; Kondrashov and Crow 1991). As described above, the mutation load is essentially determined only by the mutation rate per genome, so we would expect populations of higher ploidy to suffer from proportionally higher mutation load, even if the severity of harmful mutations differs between ploidy states. To evaluate this assumption, we need estimates of the mutation rate under alternative ploidy states that minimize other confounding effects.

Haploid and diploid mutation rates are difficult to compare using typical fluctuation tests, because a detectable phenotype in diploids generally requires both a mutation and a loss of heterozygosity at the marker locus; mutation accumulation followed by whole-genome sequencing is a more accurate approach. A recent mutation accumulation study in $S$. cerevisiae compared haploids and diploids from the same genetic background (Sharp et al. 2018). In this experiment, mutations accumulated for approximately 1600 generations in over 200 MA lines, resulting in over 2000 spontaneous mutations. Haploid and diploid lines showed similar cell division rates, but there were many differences in the number and spectrum of mutations that accumulated. The rate of single-nucleotide changes per base pair per generation was about $4.0 \times 10^{-10}$ in haploids and $2.9 \times 10^{-10}$ in diploids. The lower mutation rate per base pair in diploids means that the genome-wide mutation rate in diploids was only about 1.4-times that of haploids, rather than double. This suggests that the long term cost of diploidy relative to haploidy in terms of mutation load may be much less than is generally assumed. The flip-side is also true: when the appearance of beneficial mutations is limiting, diploids may not gain the entire 2-fold mutation rate advantage that is often predicted.

In addition to global differences in the per-base pair mutation rate, differences in the spectrum and location of nucleotide substitutions between ploidy states have also been identified. Assuming the same mutations are beneficial in different ploidy backgrounds, recessive mutations will be more accessible to haploids, where their effects are immediately "seen" by selection (this has been referred to as "Haldane's sieve", Haldane 1924, 1927). The fixation of recessive mutations in haploids but not higher ploidy levels has indeed been shown for $S$. cerevisiae evolved in different environments including rich medium (standard lab YPD; Marad, Buskirk and Lang 2018), glucose-limitation (Kvitek and Sherlock 2013; Sellis et al. 2016), the 
sub-optimal carbon raffinose (Scott et al. 2017), and fluconazole (Anderson, Sirjusingh and Ricker 2004).

It is worth noting that even fully recessive mutations can contribute to adaptation in diploids if loss of heterozygosity ( $\mathrm{LOH}$ ) at the relevant locus occurs before the mutant allele is lost; as noted above, alleles with mild effects can segregate for longer than one might intuitively expect. Initially-diploid $A$. nidulans strains that haploidized showed an immediate jump in fitness (Schoustra et al. 2007), attributed to the unmasking of recessive beneficial mutations that were already present in the diploid population. Relatively rapid LOH has been observed in an experimental context (Anderson, Sirjusingh and Ricker 2004; Gresham et al. 2008; Gerstein, Kuzmin and Otto 2014; Ratcliff et al. 2015; Sharp et al. 2018), demonstrating the important role of mitotic recombination, the primary mechanism that causes $\mathrm{LOH}$ in yeast (Hiraoka et al. 2000; St Charles et al. 2012), during evolution. As noted above, sexual reproduction can also generate homozygosity, particularly when sex occurs by selfing. While sex is relatively uncommon in S. cerevisiae, it tends to occur within tetrads (akin to selfing; Ruderfer et al. 2006, Magwene et al. 2011).

In some cases, mutations may be most beneficial in the heterozygous state, a phenomenon known as overdominance. A handful of beneficial overdominant alleles have been identified in S. cerevisiae experiments, including three CNVs that arose during evolution in glucose-limited chemostats (Sellis et al. 2016), and an AEP3 allele found to be overdominant for growth at 37 ${ }^{\circ} \mathrm{C}$ in a study of hybrids created from divergent parental strains of $S$. cerevisiae (Shapira and David 2016). Despite some additional well-supported examples from outside the fungal literature, there is little evidence so far that long-term selection favours heterozygosity at very many loci (Hedrick 2012). Still, theory suggests heterozygote advantage could be an important transient state involved in adaptation in diploids (Sellis et al. 2011).

Several other observations from the Sharp et al. (2018) study are potentially relevant to predicting how alternative ploidy states might evolve and their relative advantages in the long term. It has been thought that since diploids maintain more than one copy of each chromosome throughout the cell cycle, they may be better able to access homology-based DNA repair pathways than haploids, helping to avoid the insertion-deletion (indel) mutations that often result from alternative forms of repair (Skoneczna et al. 2015). However, haploids 
and diploids had similar rates of indel mutations, suggesting that homologous repair may not be a key feature of ploidy differences in mutation (Sharp et al. 2018).

On the other hand, large structural mutations, namely aneuploidy and segmental duplications, accumulated in diploid but not haploid MA lines (Sharp et al. 2018), suggesting that the presence of multiple chromosome copies increases the rate of spontaneous structural changes. Indeed, the data are consistent with a twofold-higher rate of chromosome gains per genome in diploids compared with haploids, which may simply reflect the fact that segregation involves twice as many chromosomes in diploids (this result refers to only to wildtype lines where the DNA recombination/repair tranlocase gene RDH54 was not deleted; $r d h 54 \Delta$ lines show a larger difference in aneuploidy rates; Sharp et al. 2018). Additional experimental studies have also found that large copy number variants (CNVs) are more likely to evolve in diploid compared to haploid populations maintained in chemostats (Gresham et al. 2008; Kvitek and Sherlock 2013; Sellis et al. 2016) and batch culture (Selmecki et al. 2015; Fisher et al. 2018) under a variety of environmental conditions. In some cases, aneuploidies have only been observed in non-haploid populations (Selmecki et al. 2015; Fisher et al. 2018). It would be valuable to systematically examine how the phenotypic effects of aneuploidy depend on the ploidy background.

Multiple experiments indicate that polyploidy (i.e., triploidy, tetraploidy, and so on) may be a particularly unstable genomic state. Although to this point we have restricted most of our discussion to large-scale ploidy changes (i.e., the influence of changing the copy number of the entire set of chromosomes), beneficial aneuploidy has been documented in many environmental conditions (reviewed in Rancati and Pavelka 2013; Todd, Forche and Selmecki 2017; Gilchrist and Stelkens 2019). If ploidy shifts influence genome stability, then the initial ploidy mutation could act as a "modifier" allele, where the ploidy change itself is not necessarily advantageous, but leads to a change in the rate of aneuploidy, increasing the rate that beneficial aneuploidy arises in the population. There is considerable evidence that increasing ploidy leads to increased rates of genome instability in S. cerevisiae (Mayer and Aguilera 1990; Andalis et al. 2004; Storchova 2014), Candida albicans (Avramovska and Hickman 2019) and Candida tropicalis (Du et al. 2018). Aneuploidy has been shown to be an important but transient state in $S$. cerevisiae populations adapting to a rapid temperature increase (Yona et al. 2012; Yona, Frumkin and Pilpel 2015) and among a series of clinical strains of Candida albicans isolated within the same patient (where aneuploidy itself was generally not beneficial, 
but may have acted as a stepping stone to the evolution of beneficial LOH; Ford et al. 2015). An elegant experiment by Harrison et al. (2014) demonstrated that ploidy variation followed by aneuploidy occurs rapidly under certain conditions; they found that exposure of Candida albicans and related species to the drug fluconazole caused some cells to become tetraploid, with aneuploids then arising following continued drug exposure.

The conclusions here are consistent with the intertwined nature of ploidy and the environment we have previously described. Differences in the patterns of adaptive evolution between ploidy states and the potential fitness consequences of transitions between states could stem partly from an effect of ploidy on the spontaneous mutation rate and spectrum. Ploidy may also affect the genomic location of mutations: Sharp et al. (2018) found that mutations in haploids lines shifted towards late-replicating regions of the genome compared to diploids. While there is much still to learn about how the mutation process might depend on ploidy, it is clear that selection and dominance are unlikely to be the only factors influencing the spectrum of mutations that become fixed in different ploidy backgrounds. In the short term, a single ploidy level may not be uniformly favoured because mutational dynamics are not uniform: when recessive mutations are required for adaptation, haploidy may be favoured; if overdominant mutations are common, diploidy may be favoured; if structural mutations are needed, they may arise more frequently or be beneficial only in higher ploidy states. As we discuss next, the rate of adaptation is influenced by nearly all of the phenomena we have just discussed.

\section{Rate of adaptation differences}

The rate of adaptation is a measurement of how rapidly an population increases in mean fitness. It is a function of how quickly beneficial mutations both arise and spread through the population, and hence is influenced by the dynamics of both mutation and selection. Some of the earliest yeast experiments to study the role of ploidy did so through the lens of adaptation rates (Paquin and Adams 1983). In large populations where mutations are not limiting, haploids are expected to adapt faster than diploids or higher-ploidy populations, if all else is equal. Indeed, multiple experiments that were initiated from isogenic ancestral strains found that haploid populations adapted faster than diploids in S. cerevisiae (Zeyl, Vanderford and Carter 2003; Gerstein et al. 2011; including in haploid-founded populations that eventually autodiploidize Fisher et al. 2018), and Aspergillus nidulans (Schoustra et al., 2006). At smaller population sizes, where the supply of mutations is more limited, there appears to be little difference in rates of adaptation (Zeyl et al., 2003). By contrast, in initially-diverse strains 
(including outcrossed heterozygous diploids), where evolution can use standing genetic variation in addition to de novo mutations, there is evidence that diploids adapt faster than haploids (Kosheleva and Desai 2018).

As described above, the spectrum of mutations may differ between populations of different ploidy, and both the number of mutations (the beneficial mutation rate) and the distribution of effect sizes can directly impact the rate of adaptation. In raffinose, tetraploid populations have been found to have a broader distribution of beneficial mutations (Scott et al. 2017), and to evolve faster than diploid or haploid populations (Selmecki et al. 2015). The effect size of alleles as mediated by dominance has also been shown to drive differences in the rate of adaptation. At high concentrations of fluconazole, where dominant or semi-dominant mutations do not appear to be available, haploids adapt much faster than diploids; when populations were evolved at a concentration of fluconazole where semi-dominant mutations were previously shown to exist (Anderson et al., 2003), diploids were able to acquire mutations and adapt faster than haploids; when the diploid population size was halved or the haploid population size doubled (to hold constant the total number of chromosomes), this advantage disappeared, and mutations were acquired at the same rate (Anderson, Sirjusingh and Ricker 2004). Similarly, Thompson et al. (2006) found that mutator strains of diploid S. cerevisiae seemed to have access to strongly beneficial mutations unavailable to haploid mutators and non-mutators, although the molecular basis for this difference was not determined.

The rate of adaptation is an essential element of the evolutionary process but seems insufficient to explain all of the patterns of ploidy variation observed in vitro. In at least three environments, the ploidy state of $S$. cerevisiae that adapted fastest was overcome by slowerevolving diploids (tetraploids adapting to raffinose: Selmecki et al. 2015; haploids adapting to YPD: Gerstein et al. 2006, 2011; Fisher et al. 2018; haploids adapting to salt: Gerstein et al. 2006, 2011). This disconnect between the rate of adaptation and the drive towards baseline ploidy reinforces that caution is required when using short term patterns, such as faster adaptation in a given ploidy state, to predict long-term evolutionary outcomes.

\section{Conclusions and Future Directions}

Ploidy variation among individuals is found in fungal populations from diverse species isolated from many different ecological contexts and arises rapidly in vitro. Frequently, lab populations are observed to transition towards their baseline ploidy ("ploidy drive"). We have argued that a 
population genetics perspective can help to tease apart how multiple factors contribute to ploidy evolution. In particular, the population genetics framework underscores the need to explicitly distinguish between the rate that ploidy variants originate in populations, the rate that ploidy variants might invade populations, and the ploidy state that is the most evolutionarily stable in the long term.

Viewing empirical results through the lens of population genetics highlights that non-selective forces can be relevant to the evolution of ploidy. While microbial populations are usually relatively large, and it is tempting to think that observed dynamics are always attributable to selection, population bottlenecks and subdivision can restrict effective population sizes. This means that selection on alleles with relatively small effects--potentially including ploidy changes in many environments--may be ineffective, leaving the fate of such variants to genetic drift. Rates of spontaneous mutation between ploidy states are likely to be asymmetrical, such that observed ploidy evolution may have as much to do with mutation as with selection. Going forward, it will be essential to characterize ploidy evolution in terms of mutation, selection and drift, rather than solely in terms of adaptation. To truly understand the population genetic forces contributing to ploidy evolution, we will need more measurements of the rate of ploidy mutation and the fitness consequences of alternative ploidy states.

\section{Measuring rates of spontaneous ploidy change}

Several MA experiments have incidentally provided insight into the rate of ploidy mutation. indicating that spontaneous ploidy change occurs at different rates in different environmental conditions and genetic backgrounds, consistent with growing evidence of mutation rate variability for other types of genetic change. Few studies to date have been purposefully designed to directly address this issue, however. Experiments with few lines and many generations may be at risk of bias due to back mutation, or even saturation (i.e., ploidy changes are not observable in lines that have already changed ploidy). A larger number of generations may also increase the risk that lines will be lost due to random extinction or experimental mishaps.

An ideal MA experiment designed to estimate the rate of ploidy change would involve many lines for a moderate number of generations, with periodic assessment of ploidy. Ploidy variants seem to arise about two orders of magnitude less frequently than the genome-wide point mutation rate, and so large experiments may be required. For a given mutation rate, the overall 
"size" of an MA experiment—number of lines times number of generations—will determine the absolute number of new mutations that accumulate, and therefore the precision of the resulting mutation rate estimates. Concerning ploidy changes, For MA experiments involving sequencing, costs generally increase with the number of lines but not the number of generations; however, ploidy changes are readily assessed using less costly methods, such as flow cytometry.

\section{Measuring the intrinsic, in situ fitness effects of ploidy changes}

When ploidy variant cells arise and reach high frequencies, it is tempting to infer an immediate fitness benefit to the mutant ploidy state, which is often closer to the baseline ploidy. Alternatively, a ploidy variant might be genetically linked to other alleles that affect fitness. The ideal approach to assessing the fitness consequences of ploidy variants is a head-to-head competition experiment between the genotypes that are present in the population at the time points when the variant arises and increases in frequency. For practical reasons, this has rarely been done; it has been much more common to use fitness proxies that may not accurately capture competitive dynamics, or to conduct competition experiments against a common, marked competitor (often the ancestor). In species where cell cycles can be synchronized, such as $S$. cerevisiae, isolates of different ploidy can be directly compared using flow cytometry (e.g., Gerstein and Otto 2011). Genotypes can also be isolated and tagged with standard markers (e.g., with GFP, or auxotrophies) and then similarly compared through headto-head competition. In addition to measuring the effects of ploidy itself, more effort is needed to characterize how mutations affect fitness when they arise in alternative ploidy states of otherwise-isogenic backgrounds.

\section{Short-term versus long-term dynamics}

The presence of ploidy variation within and among natural populations demands an explanation, and experimental approaches will remain a vital part of this effort. However, laboratory studies are necessarily limited in time scale, and we should be cautious when interpreting experimental observations in the context of predictions from population genetics theory, which may not be relevant at the same time scales. For example, a transition from haploid to diploid could provide a transient evolutionary benefit by masking recessive deleterious mutations, but the mutation rate in microbial populations may be too low for this effect to be very relevant over the course of only a few hundred generations. In this context, other explanations for short-term ploidy evolution may be more useful. 
The ploidy state with the highest fitness during a short-term lab experiment may not be the most evolutionarily robust in the long term. For instance, some ploidy states may be evolutionarily unstable if they are subject to a high rate of reversion through subsequent mutation. For a given organism, the current or baseline ploidy state is not only likely to be the most evolutionarily stable, but also reveals the most likely historical context of selection, including selection on the mutation rate itself. For example, haploid populations of $S$. cerevisiae frequently show spontaneous transitions to diploidy, but in the wild-type form of this organism such transitions occur naturally and rapidly by mating-type switching and mating; we might therefore expect little historical selection to reduce the ploidy mutation rate in haploids, since this ploidy state has been rare throughout the recent evolutionary history of this organism.

Here we have argued that much can be gained from viewing ploidy variation through the lens of population genetics. This framework also highlights a number of gaps in our understanding of when and why ploidy variation is observed. We hope this will inspire microbiologists working in diverse systems and contexts to consider how population size, mutation, and selection combine to influence the evolutionary dynamics of ploidy in the field and in the lab. 


\section{Figures and Boxes}

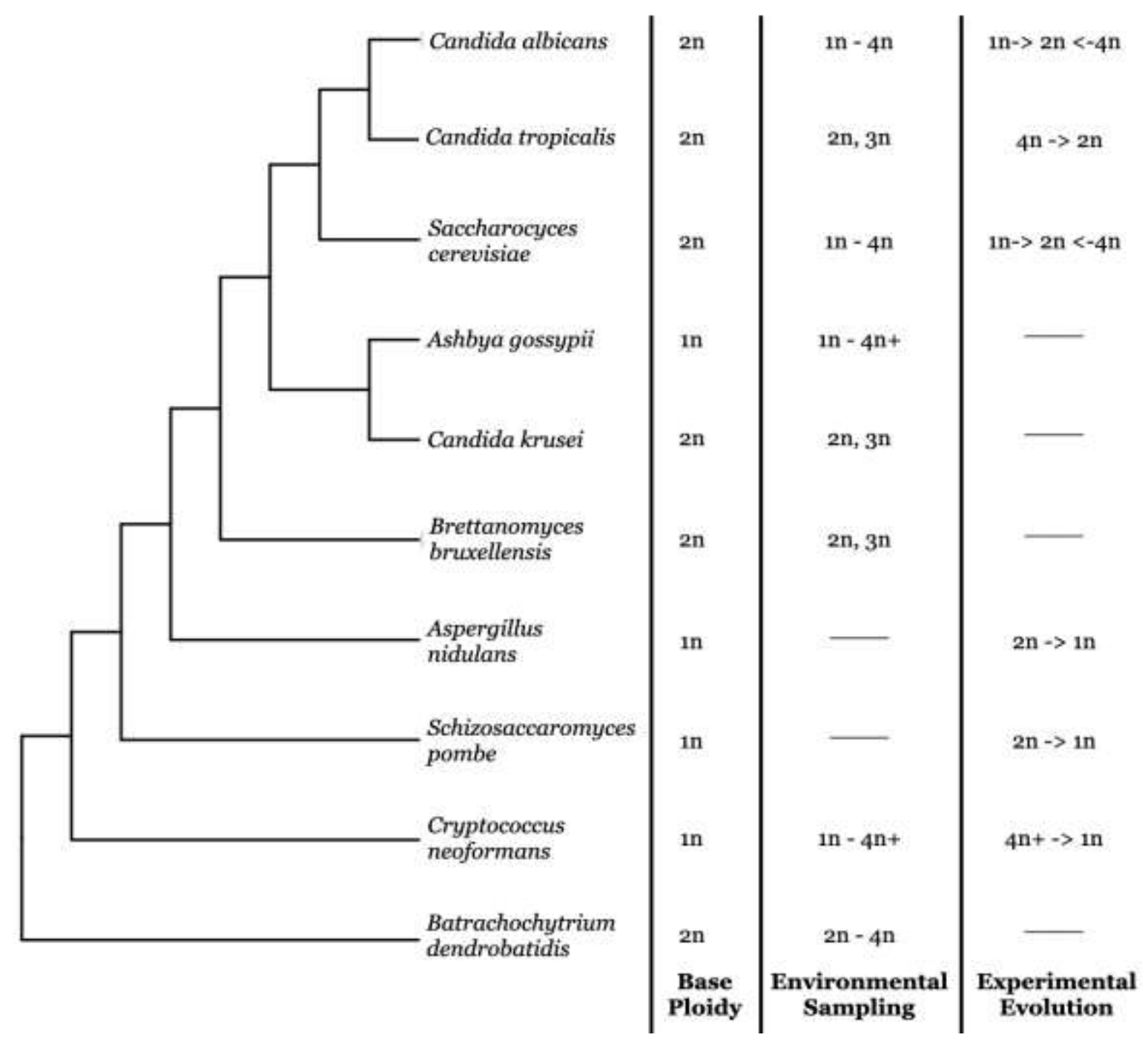

Figure 1. Within-species ploidy variation has been observed across the fungal

phylogeny. The phylogeny was made in TreeBase (Steenwyk and Rokas 2019) based on the Newick file provided by (Kiss et al. 2019). The branch lengths are not proportional to genetic relatedness. When the required species was not present in the original tree, we used a sister taxon to maintain the correct topology. The Environment column reflects ploidy variation observed among natural isolates. Experimental evolution indicates that ploidy drive towards the base genome was observed during in vitro transfer experiments conducted at a high population size where selection is expected to be effective. Horizontal lines indicate no data available (Environmental sampling), or the experiment has not been conducted (Experimental evolution), to the best of our knowledge. 


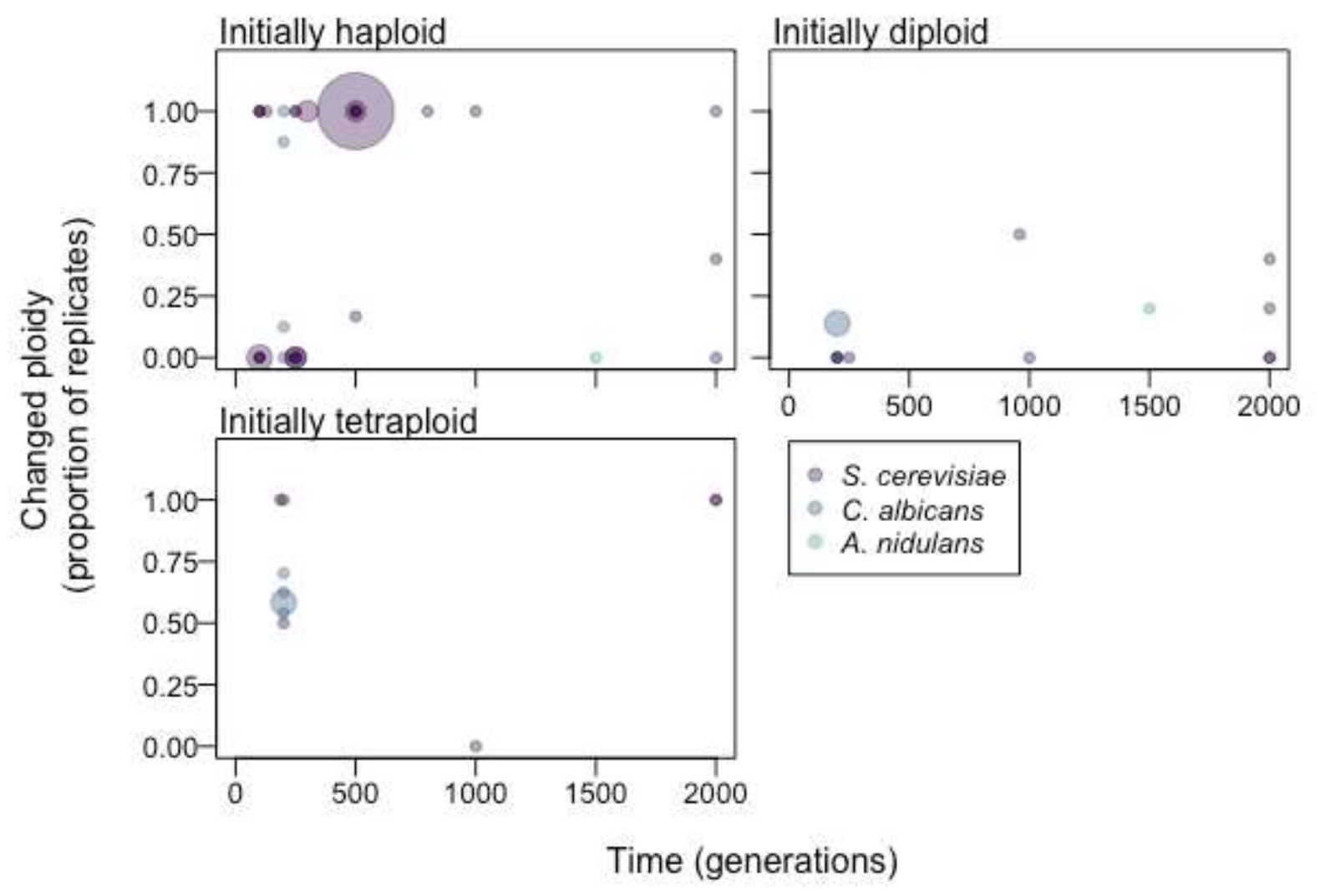

Figure 2. Initial ploidy influences the proportion of replicates that evolve an altered genome size. Each circle represents replicate lines of the same initial ploidy from one experiment. The size of the circle is proportional to the number of evolved replicate lines. Evolved ploidy was determined through flow cytometry, which is not a perfect quantification. In some cases, the evolved populations were mixed, thus when possible, the most prominent sub-population was recorded (Table S1). Only experiments that calculated the number of generations of evolution were included here; data from additional experiments are included in Table S1. 


\section{A. Chemostat culture}

Continuous liquid media replacement maintains stable population size.
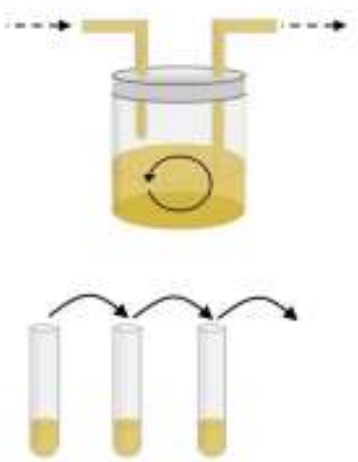
new liquid media, with moderate dilution.

C. Mutation accumulation

Frequent streaking on new plates for singlecell bottlenecks.

\section{Fluctuation test}

One-time plating on selective and nonselective media to score mutation rate at specific loci.

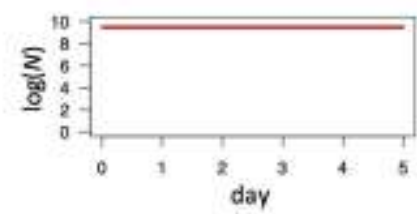

Example: $300 \mathrm{~mL}$ culture with stable population size.

$N_{q}$ : very large

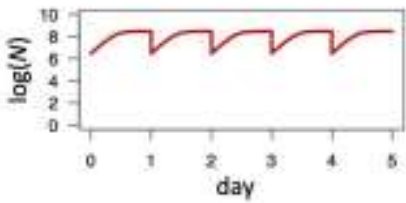

Example: logistic growth in $1 \mathrm{~mL}$ culture with daily 100 . fold dilution.

$N_{p}$ : large

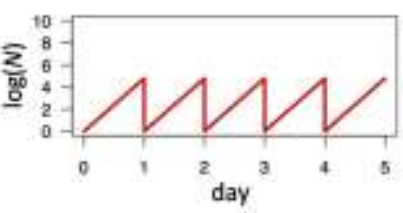

Example: exponential growth with daily streaking to start new colonies from single cells.

$N_{e}:$ very small

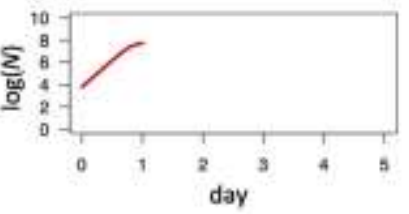

Example: exponential growth in $0.2 \mathrm{~mL}$ culture followed by plating.

$N_{e}$ : small

Figure 3. Alternative methods for studying microbial populations and implications for effective population sizes. For additional discussion of these methods see McDonald (2019), Dunham et al. (2017), and Lang (2018). 
Box 1. Elements of population genetics applied to the dynamics of ploidy change.

\begin{tabular}{|c|c|c|}
\hline $\begin{array}{l}30 \% \\
30 \% \\
30 \% \\
\text { Variants ar }\end{array}$ & $\begin{array}{l}\text { Low } \mu \text {, low } \\
\text { initial frequency } \\
\text { High } \mu \text {, high } \\
\text { initial frequency }\end{array}$ & $\begin{array}{l}N \mu \text { ploidy variants arise each generation due to } \\
\text { mutation, where } N \text { is the population size and } \mu \text { is the } \\
\text { rate of ploidy change per cell division. For most } \\
\text { mutations } \mu \text { is small, making it unlikely that more } \\
\text { than one copy will arise at a time, such that mutant } \\
\text { alleles have will initially be at frequency } 1 / N \text {. If } \\
\text { ploidy is highly mutable }(\mu>1 / N) \text {, we expect } \\
\text { multiple ploidy mutants each generation in large } \\
\text { populations, i.e., } N \mu \gg 1 \text {. If back-mutations occur at } \\
\text { rate } v \text {, the expected equilibrium frequency of a } \\
\text { selectively-neutral ploidy variant is } \mu /(\mu+v) \text {. }\end{array}$ \\
\hline Variant frequency & $\begin{array}{l}\begin{array}{l}\text { Decrease in } \\
\text { frequency } \\
\text { (loss) }\end{array} \\
\text { Increase in } \\
\text { frequency }\end{array}$ & $\begin{array}{l}\text { Alleles can become fixed or lost due to the effects of } \\
\text { random sampling in finite populations - genetic } \\
\text { drift. The probability that a selectively-neutral } \\
\text { variant will eventually become fixed is equal to its } \\
\text { initial frequency; rare variants are therefore very } \\
\text { likely to be lost by drift. Allele frequencies will drift } \\
\text { more rapidly in smaller populations, and when the } \\
\text { effective population size }\left(N_{e}\right) \text { is low. In populations } \\
\text { experiencing periodic bottlenecks, } N_{e} \text { is approxi- } \\
\text { mated by the harmonic mean population size, } \widetilde{N} \text {, } \\
\text { across } t \text { time points: } N_{e} \cong \widetilde{N}=t / \sum t \frac{1}{N_{i}} \text {. }\end{array}$ \\
\hline C. Selectic & $\begin{array}{l}\text { Beneficial } \\
\text { variants }\end{array}$ & $\begin{array}{l}\text { Beneficial mutations will not necessarily escape the } \\
\text { effects of drift. In fact, in the simplest case, a single- } \\
\text { copy variant that confers a fitness benefit } s \text { has a } \\
\text { probability of just } 2 s \text { of eventual fixation. In } \\
\text { fluctuating populations this prediction is reduced to } \\
2 s(\widetilde{N} / \bar{N}) \text {, where } \bar{N} \text { is the arithmetic mean pop- } \\
\text { ulation size (Ewens, 1967). However, these simple } \\
\text { models fail to capture several characteristics of } \\
\text { microbial populations, including overlapping } \\
\text { generations and a non-Poisson distribution of } \\
\text { offspring numbers (Patwa \& Wahl, 2008). }\end{array}$ \\
\hline
\end{tabular}




\section{Box 2. Simulated dynamics of beneficial ploidy variants.}

To illustrate the factors that would affect the evolution of intrinsically-beneficial ploidy shifts in laboratory cultures, we simulated asexual populations undergoing logistic growth with periodic dilutions. In each simulation, an initial population of $10^{4}$ cells grows at rate $r=0.4$ per hour with carrying capacity $K$. In each cell division, a ploidy variant arises with probability $\mu$, with no back mutation, and confers a fitness effect $s$. Every day the population is diluted by factor $d$, with cells sampled at random. For each parameter set of interest, we simulated 500 populations evolving for 60 transfers (days). Plotted is the number of transfers elapsed before the ploidy variant reached a frequency of 0.95 or higher ("fixation"), averaged over replicate simulations. Values of "60+" indicate that the average time to fixation was longer than 60 days. Note that these simulations consider the time to fixation of ploidy variants that are identical by state, not identical by descent. In other words, multiple ploidy variants that arise independently by mutation can contribute to fixation.

These simulations represent a simplified case, but they illustrate several dynamics relevant to the spread of ploidy variants.

Naturally, ploidy variants with more-beneficial effects are expected to become fixed more rapidly. When the beneficial effect is mild (e.g., on the order of $0.1 \%$ ), a ploidy variant may not become fixed rapidly even in the presence of a high mutation rate. It is also evident that higher mutation rates accelerate fixation; high mutation rates will both reduce the waiting time for the first ploidy variant to arise and provide upward pressure on the variant frequency, helping positive selection overcome the effects of drift. Larger populations (higher $K$ ) also favour the fixation of ploidy variants. The expected time to fixation of a given beneficial allele that starts as a single copy scales log-linearly with $N\left(t_{\text {fixaton }} \cong 2 \ln (N\right.$ $-1) / s$, Otto and Whitlock 2005), because an allele will take longer to spread through a larger population. In our simulations, multiple mutants can coexist, and the rate of introduction of the ploidy variant scales linearly with $N$, overwhelming the effect of $N$ on time to fixation. Stronger dilutions (lower $d$ ), as well as lower $K$, will reduce the effective population size and increase the effect of drift, leading to longer fixation times.

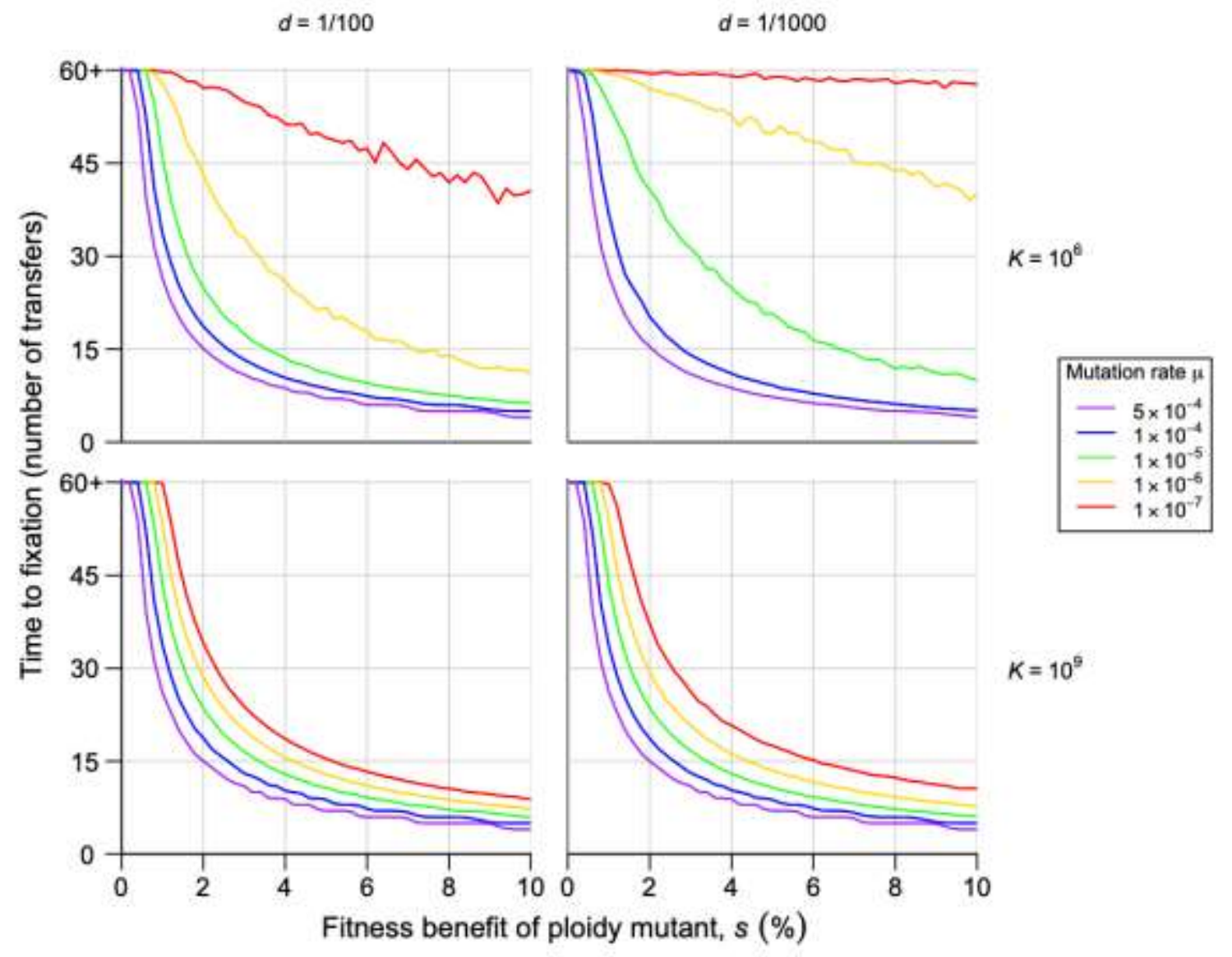




\section{Tables}

Table 1. Frequency of ploidy variation in Saccharomyces cerevisiae isolate collections

\begin{tabular}{|c|c|c|c|c|c|}
\hline Reference & Origin & Haploid & Diploid & Triploid & Tetraploid + \\
\hline Ezov 2006 & Wild (Israel) & 0 & 14 & 10 & 58 \\
\hline Gallone 2016 & Beer & 0 & 14 & 10 & 58 \\
\hline Gallone 2016 & Bread & 0 & 2 & 0 & 2 \\
\hline Gallone 2016 & Spirits & 0 & 5 & 1 & 3 \\
\hline Gallone 2016 & Sake & 0 & 5 & 1 & 0 \\
\hline Albertin 2009 & Food-processing & 0 & 16 & 0 & 10 \\
\hline Duan 2018 & Wild \& Domesticated (China) & 12 & 233 & 20 & 1 \\
\hline Peter 2018 & Geographically diverse & 9 & 694 & 47 & 44 \\
\hline Zhu 2016 & Mostly clinical & 16 & 82 & 23 & 23 \\
\hline \multirow[t]{2}{*}{ Al Safadi 2010} & Pearl millet beer & 0 & 0 & 0 & 6 \\
\hline & Total & $3 \%$ & $78 \%$ & $8 \%$ & $11 \%$ \\
\hline
\end{tabular}




\section{Acknowledgements}

We thank Sarah (Sally) Otto for thoughtful comments and Anna Selmecki and Anja Forche for an initial spark that led to this manuscript.

\section{References}

Agrawal AF, Whitlock MC. Inferences about the distribution of dominance drawn from yeast gene knockout data. Genetics 2011;187:553-66.

Albertin W, Marullo P. Polyploidy in fungi: evolution after whole-genome duplication. Proc Biol Sci 2012;279:2497-509.

Albertin W, Marullo P, Aigle M et al. Evidence for autotetraploidy associated with reproductive isolation in Saccharomyces cerevisiae: towards a new domesticated species. J Evol Biol 2009;22:2157-70.

Al Safadi R, Weiss-Gayet M, Briolay J et al. A polyploid population of Saccharomyces cerevisiae with separate sexes (dioecy). FEMS Yeast Res 2010;10:757-68.

Andalis AA, Storchova Z, Styles C et al. Defects arising from whole-genome duplications in Saccharomyces cerevisiae. Genetics 2004;167:1109-21.

Anderson $\mathrm{CA}$, Roberts $\mathrm{S}$, Zhang $\mathrm{H}$ et al. Ploidy variation in multinucleate cells changes under stress. Mol Biol Cell 2015;26:1129-40.

Anderson JB, Sirjusingh C, Parsons AB, et al. Mode of selection and experimental evolution of antifungal drug resistance in Saccharomyces cerevisiae. Genetics 2003;163:1287-1298.

Anderson JB, Sirjusingh C, Ricker N. Haploidy, diploidy and evolution of antifungal drug resistance in Saccharomyces cerevisiae. Genetics 2004;168:1915-23.

Avramovska O, Hickman MA. The magnitude of Candida albicans stress-induced genome instability results from an interaction between ploidy and antifungal drugs. G3 2019;9:4019-27.

Barchenger DW, Lamour KH, Sheu Z-M et al. Intra- and intergenomic variation of ploidy and clonality characterize phytophthora capsici on Capsicum sp. in Taiwan. Mycol Prog 2017;16:955-63.

Berger D, Stångberg J, Grieshop K et al. Temperature effects on life-history trade-offs, germline maintenance and mutation rate under simulated climate warming. Proc Biol Sci 2017;284, DOI: 10.1098/rspb.2017.1721.

Bennett RJ, Johnson AD. Completion of a parasexual cycle in Candida albicans by induced chromosome loss in tetraploid strains. EMBO J 2003;22:2502-15.

Böndel KB, Kraemer SA, Samuels T et al. Inferring the distribution of fitness effects of spontaneous mutations in Chlamydomonas reinhardtii. PLoS Biol 2019;17:e3000192.

Borneman AR, Zeppel R, Chambers PJ et al. Insights into the Dekkera bruxellensis genomic landscape: comparative genomics reveals variations in ploidy and nutrient utilisation potential amongst wine isolates. PLoS Genet 2014;10:e1004161.

Brandt ME, Lockhart SR. Recent taxonomic developments with Candida and other opportunistic yeasts. Curr Fungal Infect Rep 2012;6:170-7.

Brewer BJ, Chlebowicz-Sledziewska E, Fangman WL. Cell cycle phases in the unequal mother/daughter cell cycles of Saccharomyces cerevisiae. Mol Cell Biol 1984;4:25292531.

Buskirk SW, Rokes AB, Lang GI. Adaptive evolution of a rock-paper-scissors sequence along a direct line of descent. bioRxiv 2019:700302.

Campbell MA, Ganley ARD, Gabaldón T et al. The case of the missing ancient fungal polyploids. Am Nat 2016;188:602-14.

Carlson MO, Gazave E, Gore MA et al. Temporal genetic dynamics of an experimental, 
biparental field population of Phytophthora capsici. Front Genet 2017;8:26.

Carreté L, Ksiezopolska E, Pegueroles $\mathrm{C}$ et al. Patterns of genomic variation in the opportunistic pathogen Candida glabrata suggest the existence of mating and a secondary association with humans. Current Biology 2018;28:15-27.e7.

Chain FJJ, Flynn JM, Bull JK et al. Accelerated rates of large-scale mutations in the presence of copper and nickel. Genome Res 2019;29:64-73.

Chang S-L, Leu J-Y. A tradeoff drives the evolution of reduced metal resistance in natural populations of yeast. PLoS Genet 2011;7:e1002034.

Charlesworth $\mathrm{D}$, Willis $\mathrm{JH}$. The genetics of inbreeding depression. Nat Rev Genet 2009;10:783-96.

Cibrario A, Avramova M, Dimopoulou M et al. Brettanomyces bruxellensis wine isolates show high geographical dispersal and long remanence in cellars. bioRxiv 2019:763441.

Cooper VS. Experimental evolution as a high-throughput screen for genetic adaptations. mSphere 2018;3:e00121-18.

Crow JF, Kimura M. Evolution in sexual and asexual populations. Am Nat 1965;99:439-50.

Daggett SS, Knighton JE, Therrien CD. Polyploidy among isolates of Phytophthora infestans from Eastern Germany. J Phytopathol 1995;143:419-22.

Dambuza IM, Drake T, Chapuis A et al. The Cryptococcus neoformans Titan cell is an inducible and regulated morphotype underlying pathogenesis. PLOS Pathog 2018;14:e1006978.

Davoli $\mathrm{T}$, de Lange $\mathrm{T}$. The causes and consequences of polyploidy in normal development and cancer. Annu Rev Cell Dev Biol 2011;27:585-610.

Dickinson WJ. Synergistic fitness interactions and a high frequency of beneficial changes among mutations accumulated under relaxed selection in Saccharomyces cerevisiae. Genetics 2008;178:1571-8.

Douglass AP, Offei B, Braun-Galleani $S$ et al. Population genomics shows no distinction between pathogenic Candida krusei and environmental Pichia kudriavzevii: One species, four names. PLoS Pathog 2018;14:e1007138.

Duan S-F, Han P-J, Wang Q-M et al. The origin and adaptive evolution of domesticated populations of yeast from Far East Asia. Nat Commun 2018;9:2690.

Du H, Zheng Q, Bing J et al. A coupled process of same- and opposite-sex mating generates polyploidy and genetic diversity in Candida tropicalis. PLoS Genet 2018;14:e1007377.

Dunham MJ, Kerr EO, Miller AW, et al.. Chemostat culture for yeast physiology and experimental evolution. Cold Spring Harb Protoc 2017; doi:10:1101/pdb.top077610.

Dyląg M, Colon-Reyes RJ, Kozubowski L. Titan cell formation is unique to Cryptococcus species complex. Virulence 2020;11:719-29.

Edgar BA, Zielke N, Gutierrez C. Endocycles: a recurrent evolutionary innovation for postmitotic cell growth. Nat Rev Mol Cell Biol 2014;15:197-210.

Ewens WJ. The probability of survival of a new mutant in a fluctuating environment. Heredity 1967;22:438-43.

Eyre-Walker A, Keightley P. The distribution of fitness effects of new mutations. Nat Rev Genet 2007;8:610-8.

Ezov TK, Boger-Nadjar E, Frenkel Z et al. Molecular-genetic biodiversity in a natural population of the yeast Saccharomyces cerevisiae from "Evolution Canyon": microsatellite polymorphism, ploidy and controversial sexual status. Genetics,2006,174:1455-68.

Farrer RA, Henk DA, Garner TWJ et al. Chromosomal copy number variation, selection and uneven rates of recombination reveal cryptic genome diversity linked to pathogenicity. PLoS Genet 2013;9:e1003703.

Filteau M, Hamel V, Pouliot M-C et al. Evolutionary rescue by compensatory mutations is 
constrained by genomic and environmental backgrounds. Mol Syst Biol 2015;11:832. Fisher KJ, Buskirk SW, Vignogna RC et al. Adaptive genome duplication affects patterns of molecular evolution in Saccharomyces cerevisiae. PLoS Genet 2018;14:e1007396.

Forche A, Magee PT, Selmecki A, et al. Evolution in Candida albicans populations during a single passage through a mouse host. Genetics 2009;182:799-811.

Forche A, Cromie G, Gerstein AC et al. Rapid phenotypic and genotypic diversification after exposure to the oral host niche in Candida albicans. Genetics 2018;209:725-41.

Ford CB, Funt JM, Abbey D et al. The evolution of drug resistance in clinical isolates of Candida albicans. eLife 2015;4, DOI: 10.7554/elife.00662.

Foster PL. Sorting out mutation rates. Proc Natl Acad Sci USA 1999;96:7617-7618.

Fox DT, Duronio RJ. Endoreplication and polyploidy: insights into development and disease. Development 2013;140:3-12.

Gabaldón T. Hybridization and the origin of new yeast lineages. FEMS Yeast Res 2020, DOI: 10.1093/femsyr/foaa040.

Gabaldón T, Fairhead C. Genomes shed light on the secret life of Candida glabrata: not so asexual, not so commensal. Curr Genet 2019;65:93-8.

Galitski T, Saldanha AJ, Styles CA et al. Ploidy regulation of gene expression. Science 1999;285:251-4.

Gallone B, Steensels J, Prahl T et al. Domestication and divergence of Saccharomyces cerevisiae beer yeasts. Cell 2016;166:1397-410.e16.

Gerstein AC. Mutational effects depend on ploidy level: all else is not equal. Biol Lett 2013;9:20120614.

Gerstein AC, Berman J. Candida albicans genetic background influences mean and heterogeneity of drug responses and genome stability during evolution in fluconazole. mSphere 2020;5, DOI: 10.1128/mSphere.00480-20.

Gerstein AC, Chun HJE, Grant A et al. Genomic convergence toward diploidy in Saccharomyces cerevisiae. PLoS Genet 2006;2:e145

Gerstein AC, Cleathero LA, Mandegar MA et al. Haploids adapt faster than diploids across a range of environments. J Evol Biol 2011;24:531-40.

Gerstein AC, Fu MS, Mukaremera L et al. Polyploid titan cells produce haploid and aneuploid progeny to promote stress adaptation. MBio 2015;6:e01340-15.

Gerstein AC, Kuzmin A, Otto SP. Loss-of-heterozygosity facilitates passage through Haldane's sieve for Saccharomyces cerevisiae undergoing adaptation. Nat Commun 2014;5:1-9

Gerstein AC, Lim H, Berman J et al. Ploidy tug-of-war: Evolutionary and genetic environments influence the rate of ploidy drive in a human fungal pathogen. Evolution 2017;71:102538.

Gerstein AC, McBride RM, Otto SP. Ploidy reduction in Saccharomyces cerevisiae. Biol Lett 2008;4:91-4.

Gerstein AC, Otto SP. Ploidy and the causes of genomic evolution. J Hered 2009;100:571-81.

Gerstein AC, Otto SP. Cryptic fitness advantage: diploids invade haploid populations despite lacking any apparent advantage as measured by standard fitness assays. PLoS One 2011;6:e26599.

Gilchrist C, Stelkens R. Aneuploidy in yeast: Segregation error or adaptation mechanism? Yeast 2019, DOI: 10.1002/yea.3427.

Goddard MR, Godfray HCJ, Burt A. Sex increases the efficacy of natural selection in experimental yeast populations. Nature 2005;434:636-40.

de Godoy LMF, Olsen JV, Cox J et al. Comprehensive mass-spectrometry-based proteome quantification of haploid versus diploid yeast. Nature 2008;455:1251-4.

Goffeau A, Barrell BG, Bussey H et al. Life with 6000 genes. Science 1996;274:546 - \&. 
Gorter FA, Derks MFL, van den Heuvel J et al. Genomics of adaptation depends on the rate of environmental change in experimental yeast populations. Mol Biol Evol 2017;34:2613-26.

Gray JC, Goddard MR. Sex enhances adaptation by unlinking beneficial from detrimental mutations in experimental yeast populations. BMC Evol Biol 2012;12:43.

Gregory TR, Mable BK. Chapter 8: Polyploidy in Animals. 2008:46.

Gresham D, Desai MM, Tucker CM et al. The repertoire and dynamics of evolutionary adaptations to controlled nutrient-limited environments in yeast. PLoS Genet 2008;4:e1000303.

Haldane JBS. A mathematical theory of natural and artificial selection, Part V: Selection and mutation. Proc Camb Philol Soc 1927;23:838-44.

Haldane JBS. A mathematical theory of natural and artificial selection. Part ii the influence of partial self-fertilisation, inbreeding, assortative mating, and selective fertilisation on the composition of mendelian populations, and on natural selection. Biol Rev Camb Philos Soc 1924;1:158-63.

Haldane JBS. The part played by recurrent mutation in Evolution. Am Nat 1933;67:5-19.

Haldane JBS. The effect of variation of fitness. Am Nat 1937;71:337-49.

Harari Y, Ram Y, Rappoport N et al. Spontaneous changes in ploidy are common in yeast. Curr Biol 2018;0, DOI: 10.1016/j.cub.2018.01.062.

Harris K. Evidence for recent, population-specific evolution of the human mutation rate. Proc Natl Acad Sci U S A 2015;112:3439-44.

Harrison BD, Hashemi J, Bibi M et al. A tetraploid intermediate precedes aneuploid formation in yeasts exposed to fluconazole. PLoS Biol 2014;12:e1001815.

Hawksworth DL. A new dawn for the naming of fungi: impacts of decisions made in Melbourne in July 2011 on the future publication and regulation of fungal names. IMA Fungus 2011;2:155-62.

Hedrick PW. What is the evidence for heterozygote advantage selection? Trends Ecol Evol 2012;27:698-704.

Hickman MA, Paulson C, Dudley A et al. Parasexual ploidy reduction drives population heterogeneity through random and transient aneuploidy in Candida albicans. Genetics 2015;200:781-94.

Hickman MA, Zeng G, Forche A et al. The "obligate diploid" Candida albicans forms matingcompetent haploids. Nature 2013;494:55-9.

Hill WG, Robertson A. The effect of linkage on limits to artificial selection. Genetical Research 1966;8:269-94.

Hiraoka M, Watanabe K, Umezu K et al. Spontaneous loss of heterozygosity in diploid Saccharomyces cerevisiae Cells. Genetics 2000;156:1531-48.

Hommel B, Mukaremera L, Cordero RJB et al. Titan cells formation in Cryptococcus neoformans is finely tuned by environmental conditions and modulated by positive and negative genetic regulators. PLoS Pathog 2018;14:e1006982.

Hong J, Gresham D. Molecular specificity, convergence and constraint shape adaptive evolution in nutrient-poor environments. PLoS Genet 2014;10:e1004041.

Immler S, Otto SP. The evolutionary consequences of selection at the haploid gametic stage. The American Naturalist 2018;192:241-9.

Jeffares DC, Rallis C, Rieux A et al. The genomic and phenotypic diversity of Schizosaccharomyces pombe. Nat Genet 2015;47:235-41.

Jorgensen P, Edgington NP, Schneider BL et al. The size of the nucleus increases as yeast cells grow. Mol Biol Cell 2007;18:3523-32.

Keightley PD, Otto SP. Interference among deleterious mutations favours sex and recombination in finite populations. Nature 2006;443:89-92. 
Kimura M. On the probability of fixation of mutant genes in a population. Genetics 1962;47:713-9.

Kimura M, Crow JF. The number of alleles that can be maintained in a finite population. Genetics 1964;49:725-38.

Kiss $\mathrm{E}$, Hegedüs $\mathrm{B}$, Virágh $\mathrm{M}$ et al. Comparative genomics reveals the origin of fungal hyphae and multicellularity. Nat Commun 2019;10:4080.

Knaus BJ, Tabima JF, Shakya SK et al. Genome-wide increased copy number is associated with emergence of dominant clones of the irish potato famine pathogen Phytophthora infestans. MBio 2020;11, DOI: 10.1128/mBio.00326-20.

Kondrashov AS, Crow JF. Haploidy or diploidy - which is better. Nature 1991;351:314-5.

Kosheleva K, Desai MM. Recombination alters the dynamics of adaptation on standing variation in laboratory yeast populations. Mol Biol Evol 2018;35:180-201.

Kvitek DJ, Sherlock G. Whole genome, whole population sequencing reveals that loss of signaling networks is the major adaptive strategy in a constant environment. PLoS Genet 2013;9:e1003972.

Lang Gl. Measuring mutation rates using the Luria-Delbruck fluctuation assay. In Genome Instability: Methods and Protocols. Muzi-Falconi M and Brown GW eds. Springer 2018.

Legras J-L, Ruh O, Merdinoglu D et al. Selection of hypervariable microsatellite loci for the characterization of Saccharomyces cerevisiae strains. Int J Food Microbiol 2005;102:7383.

Lenassi M, Gostinčar C, Jackman S et al. Whole genome duplication and enrichment of metal cation transporters revealed by de novo genome sequencing of extremely halotolerant black yeast Hortaea werneckii. PLoS ONE 2013;8:e71328.

Levy SF, Blundell JR, Venkataram S, et al. Quantitative evolutionary dynamics using highresolution lineage tracking. Nature 2015;519:181-186.

Lidzbarsky GA, Shkolnik T, Nevo E. Adaptive response to DNA-damaging agents in natural Saccharomyces cerevisiae populations from "Evolution Canyon", Mt. Carmel, Israel. PLoS ONE 2009;4:e5914.

Liti G. The fascinating and secret wild life of the budding yeast S. cerevisiae. Elife 2015;4, DOI: 10.7554/eLife.05835.

Liti G, Carter DM, Moses AM et al. Population genomics of domestic and wild yeasts. Nature 2009;458:337-41.

Liu $\mathrm{H}$, Zhang J. Yeast spontaneous mutation rate and spectrum vary with environment. Curr Biol 2019;0, DOI: 10.1016/j.cub.2019.03.054.

Li Y, Shen H, Zhou Q et al. Changing ploidy as a strategy: The Irish Potato Famine pathogen shifts ploidy in relation to its sexuality. Mol Plant Microbe Interact 2017;30:45-52.

Lu Y-J, Swamy KBS, Leu J-Y. Experimental evolution reveals interplay between Sch9 and polyploid stability in yeast. PLoS Genet 2016;12:e1006409.

Luria SE and Delbruck M. Mutations of bacteria from virus sensitivity to virus resistance. Genetics 1943;28:491-511.

Lynch M. Evolution of the mutation rate. Trends Genet 2010;26:345-52.

Lynch M, Sung W, Morris K et al. A genome-wide view of the spectrum of spontaneous mutations in yeast. Proc Natl Acad Sci USA 2008;105:9272-7.

Mable BK. Ploidy evolution in the yeast Saccharomyces cerevisiae: a test of the nutrient limitation hypothesis. J Evol Biol 2001;14:157-70.

Mable BK, Otto SP. Masking and purging mutations following EMS treatment in haploid, diploid and tetraploid yeast (Saccharomyces cerevisiae). Genet Res 2001;77:9-26.

Madlung A. Polyploidy and its effect on evolutionary success: old questions revisited with new tools. Heredity 2013;110:99-104. 
Marad DA, Buskirk SW, Lang Gl. Altered access to beneficial mutations slows adaptation and biases fixed mutations in diploids. Nat Ecol Evol 2018;2:882-9.

Martinez A, Aliouat EM, Standaert-Vitse A et al. Ploidy of cell-sorted trophic and cystic forms of Pneumocystis carinii. PLoS One 2011;6:e20935.

Mayer VW, Aguilera A. High levels of chromosome instability in polyploids of Saccharomyces cerevisiae. Mutat Res 1990;231:177-86.

McDonald MJ. Microbial experimental evolution - a proving ground for evolutionary theory and a tool for discovery. EMBO Rep 2019;e46992.

McDonald MJ, Rice DP, Desai MM. Sex speeds adaptation by altering the dynamics of molecular evolution. Nature 2016;531:233-6.

Mortimer RK. Radiobiological and genetic studies on a polyploid series (haploid to hexaploid) of Saccharomyces cerevisiae. Radiat Res 1958.

Mortimer RK. Evolution and variation of the yeast (Saccharomyces) genome. Genome Res 2000;10:403-9.

Muller HJ. Some Genetic Aspects of Sex. Am Nat 1932;66:118-38.

Mundkur BD. Interphase nuclei and cell sizes in a polyploid series of Saccharomyces. Experientia 1953;9:373-4.

Neumann FR, Nurse P. Nuclear size control in fission yeast. J Cell Biol 2007;179:593-600. Nieuwenhuis BPS, James TY. The frequency of sex in fungi. Philos Trans $R$ Soc Lond B Biol Sci 2016;371, DOI: 10.1098/rstb.2015.0540.

Ohno S. Evolution by Gene Duplication. New York: Springer Verlag, 1970.

Okagaki LH, Strain AK, Nielsen JN et al. Cryptococcal cell morphology affects host cell interactions and pathogenicity. PLoS Pathog 2010;6:e1000953.

Orr HA. The population genetics of speciation: the evolution of hybrid incompatibilities. Genetics 1995;139:1805-13.

Otto SP, Barton NH. The evolution of recombination: removing the limits to natural selection. Genetics 1997;147:879-906.

Otto SP, Whitlock MC. Fixation probabilities and times. Encyclopedia of Life Sciences 2005, DOI: $10.1038 / n p g . e l s .0005464$.

Otto SP, Whitton J. Polyploid incidence and evolution. Annu Rev Genet 2000;34:401-37.

Oud B, Guadalupe-Medina V, Nijkamp JF et al. Genome duplication and mutations in ACE2 cause multicellular, fast-sedimenting phenotypes in evolved Saccharomyces cerevisiae. Proc Natl Acad Sci U S A 2013;110:E4223-31.

Moran PAP. The Statistical Process of Evolutionary Theory. 1962. Clarendon Press, Oxford.

Paquin C, Adams J. Frequency of fixation of adaptive mutations is higher in evolving diploid than haploid yeast populations. Nature 1983;302:495-500.

Parekh SR, Yu S, Wayman M. Adaptation of Candida shehatae and Pichia stipitis to wood hydrolysates for increased ethanol production. Appl Microbiol Biotechnol 1986;25:300.

Patwa Z, Wahl LM. The fixation probability of beneficial mutations. J $R$ Soc Interface 2008:5:1279-89.

Peter J, De Chiara M, Friedrich A et al. Genome evolution across 1,011 Saccharomyces cerevisiae isolates. Nature 2018;556:339-44.

Pope CF, O'Sullivan DM, McHugh TD et al. A practical guide to measuring mutation rates in antibiotic resistance. Antimicrob Agents Chemother 2008;52:1209-1214.

Powell CD, Quain DE, Smart KA. The impact of media composition and petite mutation on the longevity of a polyploid brewing yeast strain. Lett Appl Microbiol 2000;31:46-51.

Ramsey J, Ramsey TS. Ecological studies of polyploidy in the 100 years following its discovery. Philos Trans R Soc Lond B Biol Sci 2014;369, DOI: 10.1098/rstb.2013.0352.

Rancati G, Pavelka N. Karyotypic changes as drivers and catalyzers of cellular evolvability: a 
perspective from non-pathogenic yeasts. Semin Cell Dev Biol 2013;24:332-8.

Ratcliff WC, Fankhauser JD, Rogers DW et al. Origins of multicellular evolvability in snowflake yeast. Nat Commun 2015;6:6102.

Rosenblum EB, James TY, Zamudio KR et al. Complex history of the amphibian-killing chytrid fungus revealed with genome resequencing data. Proc Natl Acad Sci U S A 2013;110:9385-90.

Ruderfer D, Pratt S, Seidel HS et al. Population genomic analysis of outcrossing and recombination in yeast. Nat Genet 2006;38:1077-81.

Saxena AS, Salomon MP, Matsuba C et al. Tempo, mode, and fitness effects of mutation in Caenorhabditis elegans over 400 generations of minimal selection. 2018:280826.

Schacherer J, Shapiro JA, Ruderfer DM et al. Comprehensive polymorphism survey elucidates population structure of Saccharomyces cerevisiae. Nature 2009;458:342-5.

Schmoller KM, Skotheim JM. The biosynthetic basis of cell size control. Trends Cell Biol 2015;25:793-802.

Schoustra SE, Debets AJM, Slakhorst M et al. Mitotic recombination accelerates adaptation in the fungus Aspergillus nidulans. PLoS Genet 2007;3:e68.

Schrider DR, Houle D, Lynch $M$ et al. Rates and genomic consequences of spontaneous mutational events in Drosophila melanogaster. Genetics 2013;194:937-54.

Schulze J, Sonnenborn U. Yeasts in the gut: from commensals to infectious agents. Deutsches Ärzteblatt International 2009;106:837.

Scott AL, Richmond PA, Dowell RD et al. The influence of polyploidy on the evolution of yeast grown in a sub-optimal carbon source. Mol Biol Evol 2017;34:2690-703.

Seervai RNH, Jones SK Jr, Hirakawa MP et al. Parasexuality and ploidy change in Candida tropicalis. Eukaryot Cell 2013;12:1629-40.

Sellis D, Callahan BJ, Petrov DA et al. Heterozygote advantage as a natural consequence of adaptation in diploids. Proc Natl Acad Sci U S A 2011;108:20666-71.

Sellis D, Kvitek DJ, Dunn B et al. Heterozygote advantage is a common outcome of adaptation in Saccharomyces cerevisiae. Genetics 2016;203:1401-13.

Selmecki AM, Maruvka YE, Richmond PA et al. Polyploidy can drive rapid adaptation in yeast. Nature 2015;519:349-52.

Serero A, Jubin C, Loeillet S et al. Mutational landscape of yeast mutator strains. Proc Natl Acad Sci U S A 2014;111:1897-902.

Shapira R, David L. Genes with a combination of over-dominant and epistatic effects underlie heterosis in growth of Saccharomyces cerevisiae at high temperature. Front Genet 2016;7:72.

Sharp NP, Agrawal AF. Low genetic quality alters key dimensions of the mutational spectrum. PLoS Biol 2016;14:e1002419.

Sharp NP, Otto SP. Evolution of sex: Using experimental genomics to select among competing theories. BioEssays 2016;38:751-757.

Sharp NP, Sandell L, James CG et al. The genome-wide rate and spectrum of spontaneous mutations differ between haploid and diploid yeast. Proc Natl Acad Sci U S A 2018, DOI: 10.1073/pnas.1801040115.

Sherman F. [1] Getting started with yeast. Methods in Enzymology. Vol 194. Academic Press, 1991, 3-21.

Shrestha SK, Miyasaka SC, Shintaku M et al. Phytophthora colocasiae from Vietnam, China, Hawaii and Nepal: intra- and inter-genomic variations in ploidy and a long-lived, diploid Hawaiian lineage. Mycol Prog 2017;16:893-904.

St Charles J, Hamilton ML, Petes TD. Meiotic chromosome segregation in triploid strains of Saccharomyces cerevisiae. Genetics 2010;186:537-50. 
St Charles J, Hazkani-Covo E, Yin Y et al. High-resolution genome-wide analysis of irradiated (UV and $\mathrm{y}$-rays) diploid yeast cells reveals a high frequency of genomic loss of heterozygosity (LOH) events. Genetics 2012;190:1267-84.

Stebbins GL Jr. Types of polyploids; their classification and significance. Adv Genet 1947;1:403-29.

Steenwyk JL, Rokas A. Treehouse: a user-friendly application to obtain subtrees from large phylogenies. BMC Res Notes 2019;12:541.

Storchova Z. Ploidy changes and genome stability in yeast: Ploidy and genome stability. Yeast 2014;31:421-30.

Storchová Z, Breneman A, Cande J et al. Genome-wide genetic analysis of polyploidy in yeast. Nature 2006;443:541-7.

Talbot NJ, Wayman M. Increase in ploidy in yeasts as a response to stressing media. Appl Microbiol Biotechnol 1989;32:167-9.

Thompson DA, Desai MM, Murray AW. Ploidy controls the success of mutators and nature of mutations during budding yeast evolution. Curr Biol 2006;16:1581-1590.

Todd RT, Forche A, Selmecki A. Ploidy variation in fungi: Polyploidy, aneuploidy, and genome evolution. Microbiol Spectr 2017;5, DOI: 10.1128/microbiolspec.FUNK-0051-2016.

Tracy MA, Lee MB, Hearn BL et al. Spontaneous polyploids and antimutators compete during the evolution of Saccharomyces cerevisiae mutator cells. Genetics 2020, DOI: 10.1534/genetics.120.303333.

Trevijano-Contador N, de Oliveira HC, García-Rodas R et al. Cryptococcus neoformans can form titan-like cells in vitro in response to multiple signals. PLoS Pathog 2018;14:e1007007.

Turanlı-Yıldız B, Benbadis L, Alkım C et al. In vivo evolutionary engineering for ethanoltolerance of Saccharomyces cerevisiae haploid cells triggers diploidization. J Biosci Bioeng 2017;124:309-18.

Venkataram S, Dunn B, Li Y et al. Development of a comprehensive genotype-to-fitness map of adaptation-driving mutations in yeast. Cell 2016;166:1585-96.e22.

Voordeckers K, Kominek J, Das A et al. Adaptation to high ethanol reveals complex evolutionary pathways. PLoS Genet 2015;11:e1005635.

Wang AD, Agrawal AF. DNA repair pathway choice is influenced by the health of Drosophila melanogaster. Genetics 2012;192:361-70.

Willis KJ, et al.. State of the world's fungi 2018. Report. State of the world's fungi 2018 Report 2018.

Wood TE, Takebayashi N, Barker MS et al. The frequency of polyploid speciation in vascular plants. Proc Natl Acad Sci U S A 2009;106:13875-9.

Wu C-Y, Rolfe PA, Gifford DK et al. Control of transcription by cell size. PLoS Biol 2010;8:e1000523.

Yauk C, Polyzos A, Rowan-Carroll A et al. Germ-line mutations, DNA damage, and global hypermethylation in mice exposed to particulate air pollution in an urban/industrial location. Proc Natl Acad Sci U S A 2008;105:605-10.

Yona $\mathrm{AH}$, Frumkin I, Pilpel Y. A relay race on the evolutionary adaptation spectrum. Cell 2015;163:549-59.

Yona $\mathrm{AH}$, Manor YS, Herbst $\mathrm{RH}$ et al. Chromosomal duplication is a transient evolutionary solution to stress. Proc Natl Acad Sci U S A 2012;109:21010-5.

Yoshida K, Schuenemann VJ, Cano LM et al. The rise and fall of the Phytophthora infestans lineage that triggered the Irish potato famine. Elife 2013;2:e00731.

Zadrag-Tecza R, Kwolek-Mirek M, Alabrudzińska M et al. Cell size influences the reproductive potential and total lifespan of the Saccharomyces cerevisiae yeast as revealed by the 
analysis of polyploid strains. Oxid Med Cell Longev 2018;2018:1898421.

Zaragoza O, Garcia-Rodas R, Nosanchuk JD et al. Fungal cell gigantism during mammalian infection. PLoS Pathog 2010;6.

Zeyl C, Vanderford T, Carter M. An evolutionary advantage of haploidy in large yeast populations. Science 2003;299:555-8.

Zheng D-Q, Jin X-N, Zhang $\mathrm{K}$ et al. Novel strategy to improve vanillin tolerance and ethanol fermentation performances of Saccharomycere cerevisiae strains. Bioresour Technol 2017;231:53-8.

Zhou X, Ballou ER. The Cryptococcus neoformans Titan cell: From in vivo phenomenon to in vitro model. Current Clinical Microbiology Reports 2018;5:252-60.

Zhu YO, Sherlock G, Petrov DA. Whole genome analysis of 132 clinical Saccharomyces cerevisiae strains reveals extensive ploidy variation. G3 2016;6:2421-34.

Zhu YO, Siegal ML, Hall DW et al. Precise estimates of mutation rate and spectrum in yeast. Proc Natl Acad Sci USA. 2014;111:E2310-E2318.

Zörgö E, Chwialkowska K, Gjuvsland $A B$ et al. Ancient evolutionary trade-offs between yeast ploidy states. PLoS Genet 2013;9:e1003388. 